\title{
Silica nanoparticles induce neurodegeneration-like changes in behavior, neuropathology, and affect synapse through MAPK activation
}

\author{
Ran You ${ }^{1,6}$, Yuen-Shan Ho ${ }^{2}$, Clara Hiu-Ling Hung ${ }^{1}$, Yan Liu', Chun-Xia Huang ${ }^{1}$, Hei-Nga Chan ${ }^{4}$, See-Lok Ho ${ }^{4}$, \\ Sheung-Yeung Lui ${ }^{4}$, Hung-Wing Li ${ }^{4}$ and Raymond Chuen-Chung Chang ${ }^{1,3,5^{*}}$ [D
}

\begin{abstract}
Background: Silica nanoparticles $\left(\mathrm{SiO}_{2}-\mathrm{NPs}\right)$ are naturally enriched and broadly utilized in the manufacturing industry. While previous studies have demonstrated toxicity in neuronal cell lines after $\mathrm{SiO}_{2}-\mathrm{NPs}$ exposure, the role of $\mathrm{SiO}_{2}-\mathrm{NPs}$ in neurodegeneration is largely unknown. Here, we evaluated the effects of $\mathrm{SiO}_{2}-\mathrm{NPs}$-exposure on behavior, neuropathology, and synapse in young adult mice and primary cortical neuron cultures.

Results: Male C57BL/6 N mice (3 months old) were exposed to either vehicle (sterile PBS) or fluorescein isothiocyanate (FITC)-tagged $\mathrm{SiO}_{2}$-NPs (NP) using intranasal instillation. Behavioral tests were performed after 1 and 2 months of exposure. We observed decreased social activity at both time points as well as anxiety and cognitive impairment after 2 months in the NP-exposed mice. NP deposition was primarily detected in the medial prefrontal cortex and the hippocampus. Neurodegeneration-like pathological changes, including reduced Nissl staining, increased tau phosphorylation, and neuroinflammation, were also present in the brains of NP-exposed mice. Furthermore, we observed NP-induced impairment in exocytosis along with decreased synapsin I and increased synaptophysin expression in the synaptosome fractions isolated from the frontal cortex as well as primary neuronal cultures. Extracellular signal-regulated kinase (ERK) and c-Jun N-terminal kinase (JNK) were also activated in the frontal cortex of NP-exposed mice. Moreover, inhibition of ERK activation prevented NP-mediated changes in exocytosis in cultured neurons, highlighting a key role in the changes induced by NP exposure.
\end{abstract}

Conclusions: Intranasal instillation of $\mathrm{SiO}_{2}-\mathrm{NPs}$ results in mood dysfunction and cognitive impairment in young adult mice and causes neurodegeneration-like pathology and synaptic changes via ERK activation.

Keywords: Silica nanoparticles, Neurodegeneration, Behavior, Synapse

\section{Background}

Neurodegenerative diseases, including Alzheimer's disease (AD), Parkinson's disease (PD), amyotrophic lateral sclerosis, and frontotemporal dementia (FTD), affect a large and ever-growing population globally [1]. AD, for example, is the major cause of dementia in the elderly

\footnotetext{
* Correspondence: rccchang@hku.hk

${ }^{1}$ Laboratory of Neurodegenerative Diseases, School of Biomedical Sciences, LKS Faculty of Medicine, The University of Hong Kong, Pokfulam, Hong Kong, SAR, China

${ }^{3}$ State Key Laboratory of Brain and Cognitive Sciences, The University of Hong Kong, Pokfulam, Hong Kong, SAR, China

Full list of author information is available at the end of the article
}

and is estimated to affect approximately 24 million people worldwide [2]. As this number is expected to double every 20 years until 2040, continued research to understand the causes as well as to elucidate drug targets is essential to enhance patient care and decrease the economic burden on both individuals and society.

Neurodegenerative diseases feature an assortment of clinical symptoms, including devastating changes in behavior and cognition. Collectively, neurodegenerative diseases are known to have convergent pathological markers, featured with selective dysfunction and loss of synapses and neurons, protein aggregates, and

(C) The Author(s). 2018 Open Access This article is distributed under the terms of the Creative Commons Attribution 4.0 International License (http://creativecommons.org/licenses/by/4.0/), which permits unrestricted use, distribution, and 
neuroinflammation [3-5]. These changes eventually disrupt connectivity in the neuronal circuitry of the brain $[6,7]$. In $\mathrm{AD}$ and other neurodegenerative diseases, these changes most notably affect the hippocampus and frontal cortex, which are the two major brain regions responsible for cognitive function [6]. The hippocampus mainly regulates learning and memory, while the frontal cortex regulates working memory, higher levels of intelligence, and emotions such as social interaction [6] and anxiety [8]. Therefore, the impaired structural and functional integrity of the neuronal and synaptic networks in the hippocampus and/or the frontal cortex are typically manifested as behavioral symptoms, such as progressive cognitive decline and mood dysfunction, in patients with neurodegenerative diseases [9]. While both pathological and behavioral symptoms have been widely documented, pathogenesis of many neurodegenerative disease is largely unknown and often sporadic, a characteristic that is closely related to long-term exposure to environmental risk factors [10,11]. Therefore, it is of vital importance to identify the environmental causes of neurodegeneration.

Silica nanoparticles $\left(\mathrm{SiO}_{2}-\mathrm{NPs}\right)$ are one of the most broadly exploited nanomaterials and have been utilized in a variety of industries [12-15]. They are also the most common component in a number of airborne pollutants, including mineral dust and particulate matter (PM), found in ambient air as well as circulated air in households and workplaces. Excessive exposure to $\mathrm{SiO}_{2}-\mathrm{NPs}$ has been shown to cause numerous health issues in susceptible cohorts, such as workers in industrial fields $[16,17]$. In fact, recent studies have indicated that the brain may be a potential target for $\mathrm{SiO}_{2}$-NPs. After inhalation, $\mathrm{SiO}_{2}$-NPs penetrate the epithelium of the respiratory tract and are then translocated to the brain via either the circulatory system or the olfactory nerve [18]. Once in the brain, these nanoparticles may be toxic to neurons and even affect behavior. For example, in an in vitro study using multiple neuroblastoma cell lines, $\mathrm{SiO}_{2}$-NPs dose-dependently induced cytotoxicity and AD-like pathology [19]. $\mathrm{SiO}_{2}$-NPs were also shown to induce PD-like behavioral changes in zebrafish [20]. While these findings suggest that respiratory tract exposure to $\mathrm{SiO}_{2}$-NPs could be a risk factor for neurodegeneration, this has not been fully explored, and the mechanisms underlying these effects are largely unknown.

In the present study, we evaluated the role of amorphous $\mathrm{SiO}_{2}$-NPs through respiratory tract in the development of neurodegeneration-like changes in behavior and pathology in young adult mice. We used fluorescein isothiocyanate (FITC)-tagged $\mathrm{SiO}_{2}-\mathrm{NPs} \quad\left(\mathrm{FITC}-\mathrm{SiO}_{2}-\mathrm{NPs}\right.$, $\mathrm{NP}$ ), which have similar toxicity as untagged $\mathrm{SiO}_{2}-\mathrm{NPs}$ $[21,22]$, and investigated their longitudinal effects on mood and cognition. To further investigate the mechanisms underlying the behavioral changes in the NP-exposed mice, we first examined the deposition of $\mathrm{NP}$ in the medial prefrontal cortex (mPFC) as well as the cornu ammonis area (CA)1, CA3, and dentate gyrus (DG) in the hippocampus, and then documented the histological changes in the neurons and microglia in these regions. The associated pathological changes, including hyperphosphorylated tau, synaptic endocytosis and exocytosis, and synaptic density, were also examined. The observed changes in the synapses were then confirmed with 14-day primary cultures of cortical neurons exposed to NP for $48 \mathrm{~h}$. We also studied the implication of two mitogen-activated protein kinases (MAPKs), i.e. extracellular signal-regulated kinase (ERK) and c-Jun N-terminal kinase (JNK), in the observed detrimental effects of NP on synapse. To our knowledge, this is the first detailed evaluation of the adverse effects of $\mathrm{SiO}_{2}$-NPs on behavior and in the brain, particularly with regards to the role of MAPKs activation in the NP-exposed synapse.

\section{Results}

\section{Characterization of the FITC-SiO ${ }_{2}$-NPs}

The morphology and size of the NP were characterized by transmission electron microscopy (TEM) (Fig. 1a). According to our data, these NP have an average diameter of $115 \mathrm{~nm}$ (Fig. 1b). Since the NP used in this study were suspended in aqueous fluids, namely phosphate buffered saline (PBS) and cortical neuron cell culture medium, we evaluated their hydrodynamic properties in these two fluids as well as water using dynamic light scattering (DLS) (Fig. 1c). The mean hydrodynamic diameter of the NP in water was $159.8 \mathrm{~nm}$, while the zeta potential was $-51 \mathrm{mV}$. Notably, the NP had a similar hydrodynamic diameter and zeta potential in both PBS and in culture medium compared to that observed in water.

\section{FITC-SiO ${ }_{2}-\mathrm{NPs}$ decreased social interaction activity, induced anxiety and cognitive impairment}

Motor function, mood, and cognition of mice exposed to NP or PBS (control) were tested in a battery of behavioral tests (outlined in Fig. 2a) after 1 and 2 months.

\section{Locomotor and motor function}

By measuring the distance the mice traveled in an open field test, we found that the locomotor function of the $\mathrm{NP}$-exposed mice was not significantly different from that of the control mice at either time point (Additional file 1: Figure S1a). Furthermore, we also observed that the motor function of the NP-exposed mice, determined by measuring the time spent on an accelerating rotarod, was not significantly different from that of the control mice (Additional file 1: Figure S1b). These results indicated that the NP-exposed mice were as 


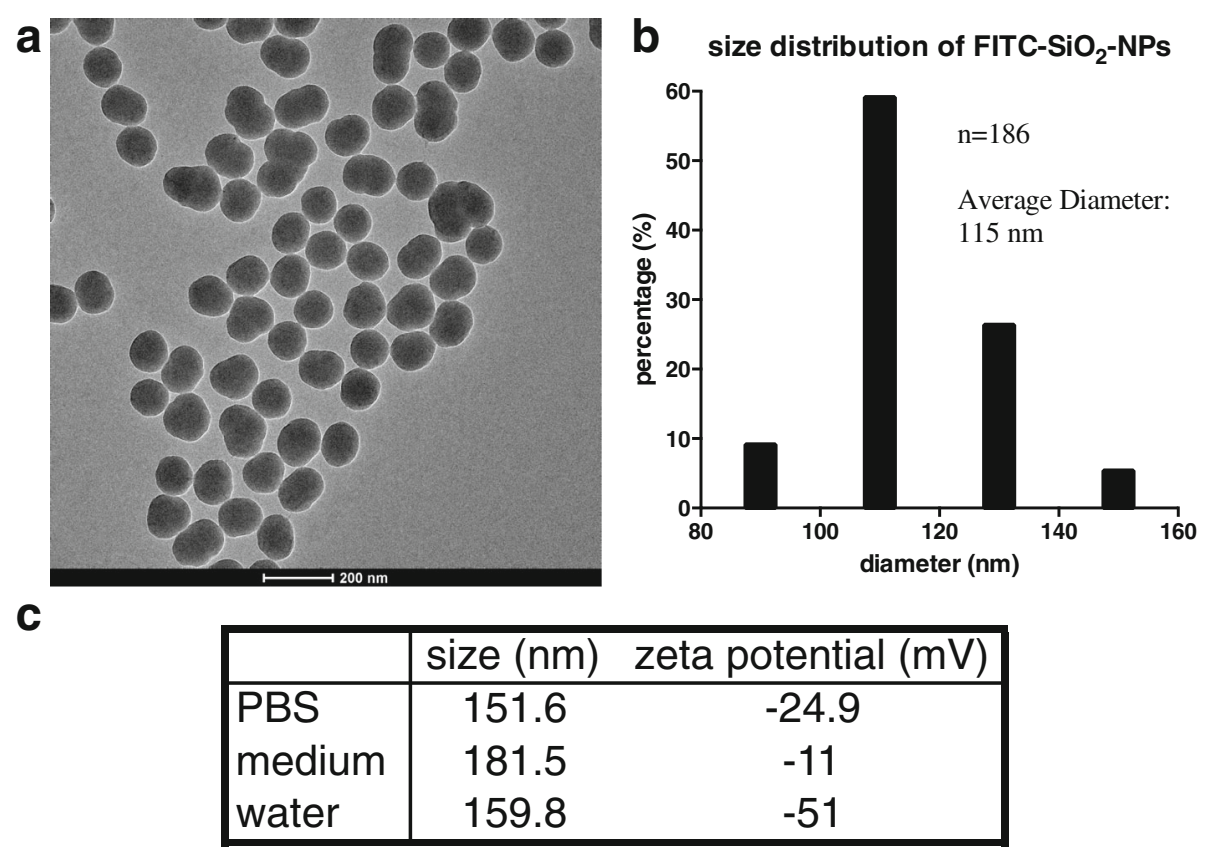

Fig. 1 Characterization of FITC-SiO ${ }_{2}$-NPs via TEM $(\mathbf{a}, \mathbf{b})$ and DLS (c). a Representative TEM image of the constructed NPs. Scale bar: $200 \mathrm{~nm}$. b Size distribution of the NPs. A total of 186 particles were measured in the TEM images. c Hydrodynamic size and zeta potential of the NP in PBS, culture medium, and water as determined by DLS

physically capable as the control mice. Thus, all of the mice, regardless of treatment, could accomplish the behavioral tests without any physical bias.

\section{Mood}

After both 1 month and 2 months of NP exposure, the mice showed a significant decrease in social interaction activity compared to the controls, as highlighted by results of the three-chambered social interaction test (1 month: -3.57 fold, $p=0.0120 ; 2$ months: -11.06 fold, $p<0.0001$; Fig. 2b). These data indicated that NP could be causing mood dysfunction in the exposed mice. Notably, anxiety and depression both involve social activity dysfunction and are common mood disorders observed in patients with neurodegeneration [23]. Therefore, we also investigated the effects of NP exposure on anxiety and depression using the elevated plus maze test and two despair tests, i.e. the forced swimming test and tail suspension test, respectively. In the elevated plus maze test, we found that the mice showed anxious behavior after 2 months of NP exposure (2 months: $-48.26 \%$, $p=0.0163$; Fig. 2c), but not at the earlier time point (Fig. 2c). Interestingly, similar trend was found in the open field test, which indicated anxiety by measuring the time spent in the central area. However, the difference between the two groups at 2 months time point was not statistically significant (Additional file 1: Figure S1c). During the despair tests, no significant differences were observed in immobility time during the forced swimming test (Additional file 1: Figure S1d) or the tail suspension test (Additional file 1: Figure S1e), suggesting that NP did not induce depression in mice.

\section{Cognition}

In the novel object recognition test, we found that the mice did not display any cognitive impairment after 1 month of NP exposure (Fig. 2d), but showed significantly impaired short-term memory after 2 months of exposure ( -1.044 fold, $p=0.0154$; Fig. 2e), which was highlighted by their failure to recognize a novel object. Consistent with these findings, in the Morris water maze test, the mice exposed to NP for 1 month did not display any significant changes in learning or memory (Fig. $2 \mathrm{f} \& \mathrm{~g}$ ). However, after 2 months of NP exposure, the mice showed impaired spatial learning and memory in the Morris water maze test, which was demonstrated by their longer escape latency in the hidden-platform training phase (repeated measures two-way ANOVA, time factor: $\mathrm{F}(4,44)=106.8, p$ $<0.0001$; treatment factor: $\mathrm{F}(1,11)=5.890, p=0.0336$; interaction: $\mathrm{F}(4,44)=0.7856, p=0.5407$; Fig. $2 \mathrm{~h}$ ) as well as the fewer number of returns to the original platform position during the probe test $(-2.417$ times, $p=0.0476$, Fig. 2i). Notably, we did not observe a significant difference in quadrate preference during the probe test (Additional file 1: Figure S1f \& g), indicating that both the control and NP-exposed groups searched for the platform using spatial memory rather than associative memory. 
a

$\mathrm{n}=8$

exposure

day

R

$\mathrm{n}=12$

exposure

day

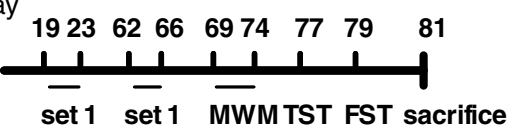

b

social interaction

(preference to helper, $n=17-20$ )

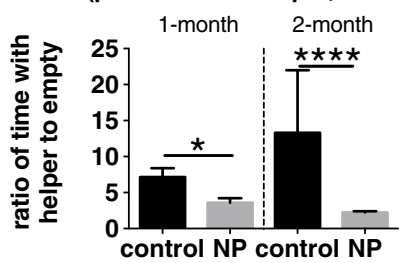

C

elevated plus maze

(time in open arm/close arm, $n=20$ )

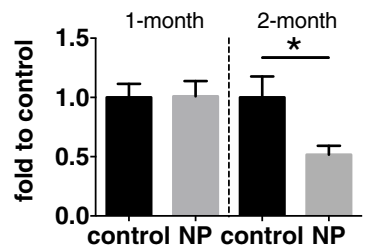

Novel object recognition test
(preference to the novel object,

Novel object recognition test

1-month exposure, $n=12$ )

2-month exposure, $n=20$ )
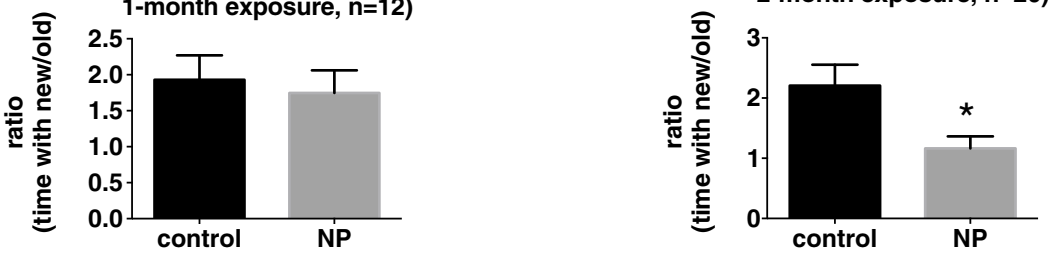

f

Morris water maze hidden platform training phase (escape latency, 1-month exposure, $n=8$ )

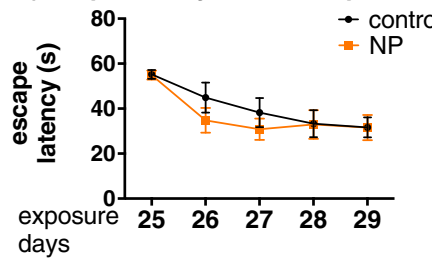

g

Morris water maze

9 probe test phase platform-crossing times

(1-month exposure, $n=6)$

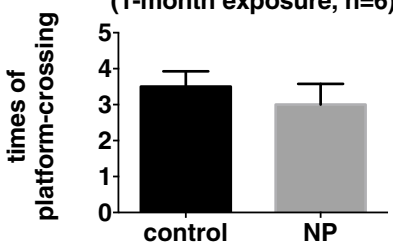

h Morris water maze hidden platform training phase (escape latency,2-month exposure, $n=12$ )

Morris water maze probe test phase platform-crossing times (2-month exposure, $n=12$ )
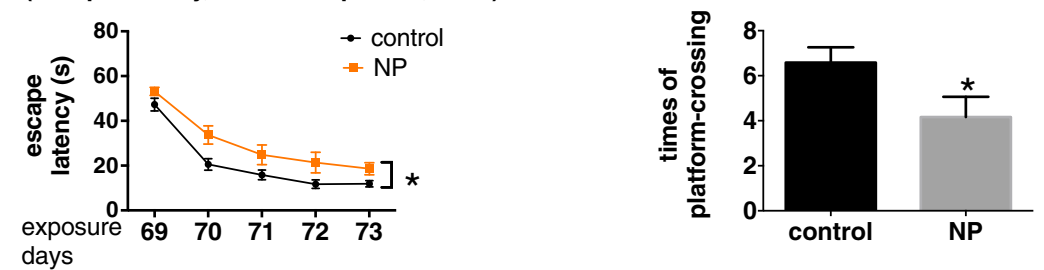

Fig. 2 (See legend on next page.) 
(See figure on previous page.)

Fig. 2 Effects of NP on mood and cognition in mice. a Experimental design for NP administration and behavioral tests. Male C57BL/6 N mice (3 months old) were administered PBS or NP (final dosage $8 \mathrm{mg} / \mathrm{kg}$ ) via intranasal instillation once per day for 81 days. Behaviors were tested according to the plan. Abbreviations: R, rotarod test; OF, open field test; EPM, elevated plus maze test; NOR, novel object recognition test; $\mathrm{SI}$, social interaction test; MWM, Morris water maze test; TST, tail suspension test; FST, forced swimming test. b SI activity (ratio of juvenile helper to empty chamber interaction, $T_{\text {helper }}\left(T_{\text {empty }}\right)$ was tested at days 23 (1 month time point, left) and 66 ( 2 month time point, right) and analyzed with an unpaired Student's t-test and unpaired non-parametric Mann-Whitney test, respectively. c EPM was used to evaluate anxiety, presented as the ratio of time spent in open arms to closed arms ( $\left.T_{\text {open }} / T_{\text {close }}\right)$, at days 19 (1 month time point, left) and 62 (2 month time point, right) and analyzed with unpaired Student's t-tests. The effects of NP exposure on cognition were tested with the NOR (d, 1 month; e, 2 months) and the MWM ( $\mathbf{f}$ and $\mathbf{g}$, training and probe phase at 1 month; $\mathbf{h}$ and $\mathbf{i}$, training and probe phase at 2 months). For the NOR, memory is demonstrated by the ratio of time interacting with new object to that interacting with the old object ( $T_{\text {new }} / T_{\text {old }}$ ). Data from 1 month and 2 months were analyzed by unpaired Student's t-tests, and unpaired Mann-Whitney test, respectively. For the MWM, the learning curve was plotted using the average escape latency per day in the training phase and was analyzed using a repeated measures two-way ANOVA. Spatial memory, represented by the platform-crossing time during the probe phase, was analyzed using unpaired Mann-Whitney tests. ${ }^{*} p<0.05$, ${ }^{* * * *} p<0.0001$, compared to controls. $n=8,12$, or 20 , indicates that each group has the same number of mice, while $n=17-20$ means that all groups included 20 mice except the 1 month control group which only had 17 as 3 videos were accidentally lost

\section{Localization of FITC-SiO ${ }_{2}$-NPs in the brain}

Using immunofluorescent staining, we observed NP deposition in the mPFC (Fig. 3a and b) as well as the CA1 and CA3 in the hippocampus, but not in the DG (Fig. 3a).

\section{FITC-SiO 2 -NPs induced neurodegeneration-like} pathological changes in the MPFC and the hippocampus Nissl staining mainly reveals the soma of neurons, including the nucleoli and the rough endoplasmic reticulum which is also called Nissl bodies. In neurodegeneration, the morphology of neurons may change, featured with the diminish of Nissl bodies. Neurons in the mPFC of the control group showed normal morphology with the nucleus in the center and Nissl bodies in purple distributed in soma, whilst neurons in the NP group showed eccentric nucleus and profound lighter staining in the soma, indicating reduction and disperse of the Nissl bodies (Fig. 4a). However, no significant differences in the Nissl bodies were observed between the control and NP-exposed groups in any region of the hippocampus (Fig. 4a).
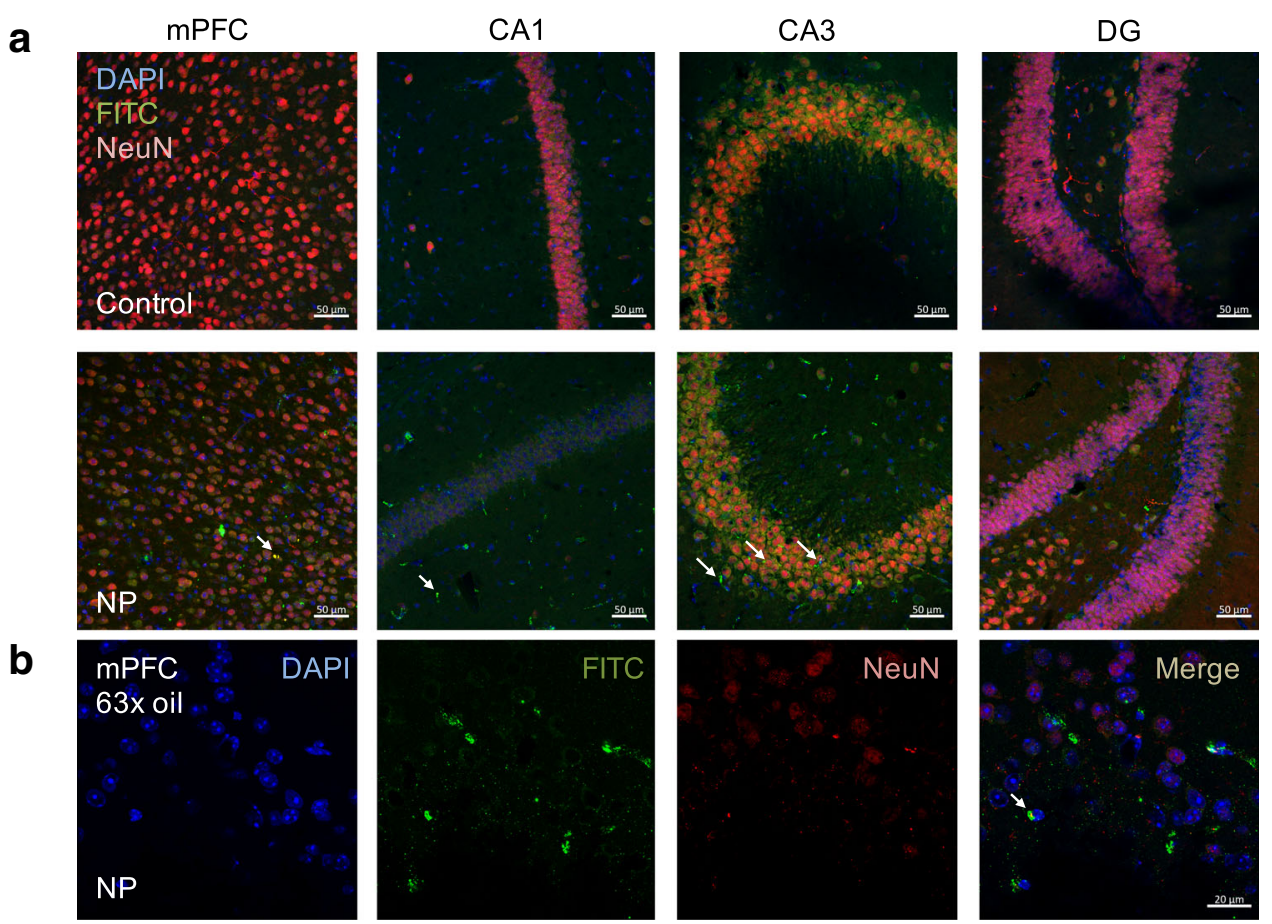

Fig. 3 Deposition of NP in the brain. After the mice were intranasally instilled with either vehicle (sterile PBS, control group) or NPs in PBS (8 mg/ $\mathrm{kg}$, NP group) once per day for 81 days, the mice were sacrificed and the brains were harvested, fixed, sectioned, and stained with anti-FITC antibody. Three non-overlapping fields in the mPFC as well as the CA1, CA3, and DG in the hippocampus were then imaged using confocal microscopy. a Representative images of the MPFC, CA1, CA3, and DG in the control group (upper panel) and NP group (middle panel) taken with a 20X objective lens. Scale bar: $50 \mu \mathrm{m}$. b Representative images of the mPFC in the NP group taken with a 63X oil lens. Scale bar: $20 \mu \mathrm{m}$. NP are highlighted by white arrows 


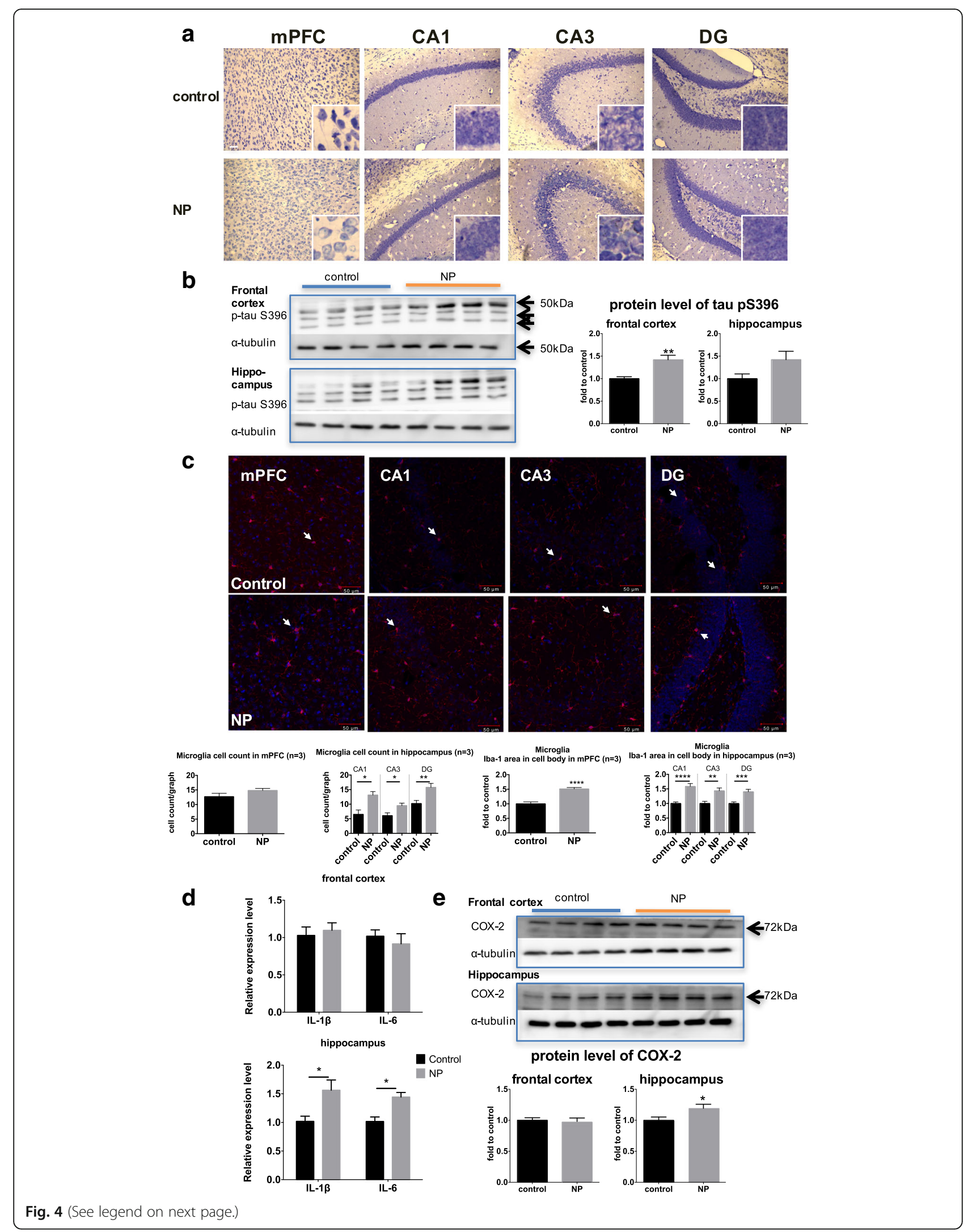


(See figure on previous page.)

Fig. 4 Neuropathological effects of NP in the brain. After the mice were intranasally instilled with either vehicle (sterile PBS, control group) or NP in PBS ( $8 \mathrm{mg} / \mathrm{kg}$, NP group) once per day for 81 days, the mice were sacrificed and the brains were harvested. Half of the brain was fixed, sectioning, and used in Niss1 staining or Iba-1 immunofluorescent staining, while the frontal cortex and the hippocampus of the other half were homogenized. a Representative images of Nissl staining in the MPFC and the hippocampus. Zoom-in image demonstrating typical morphology in Nissl staining were presented in the bottom right corner of each image ( $n=4$ per group for $\mathrm{mPFC}, n=3$ per group for hippocampus). Scale bar: $50 \mu \mathrm{m}$. b Western blot analysis of tau phosphorylation at Serine 396 in the frontal cortex and hippocampus. Image shows four representative protein bands. Band intensity was normalized to that of a-tubulin and analyzed using unpaired Student's $t$-tests ( $n=8$ for each group, except $n=7$ for hippocampus control). c Representative images of Iba-1 (red) co-stained with DAPI (blue) in the mPFC and the hippocampus. Typical microglial morphology is highlighted with an arrow. The number and area of the cell bodies with high expression of Iba-1 in each sub-region (area of interest) were measured using Image $J$ and analyzed using an unpaired Mann-Whitney test and an unpaired Student's t-test, respectively ( $n=3$ per group). Scale bar: $50 \mu m$. $\mathbf{d}$ mRNA expression of cytokines in the frontal cortex and the hippocampus by real time-PCR. Unpaired Student's $t$-test was used as statistical analysis in each marker. ( $n=6$ per group) (e) Western blot analysis of COX-2 expression in the frontal cortex and hippocampus. Image shows four representative protein bands. Band intensity was normalized to that of a-tubulin and analyzed using unpaired Student's $t$-tests $(n=8$ for each group, except $n=7$ for hippocampus control). For all analyses, ${ }^{*} p<0.05,{ }^{* *} p<0.01,{ }^{* * *} p<0.001,{ }^{* * *} p<0.0001$, compared to the controls

To further evaluate the pathology induced by NP treatment, we studied tau phosphorylation. Tau is a microtubule-associated protein that binds to and stabilizes microtubules. Moreover, increased tau phosphorylation is involved in the pathology of many neurodegenerative diseases, including $\mathrm{AD}$ and PD-related dementia [4]. Serine 396 is a common phosphorylation site in the tau protein that has been associated with neurodegeneration. In this study, tau phosphorylation at serine 396 was significantly increased in the frontal cortex of NP-exposed mice compared to the controls (41.84\%, $p=0.0019$; Fig. 4b). However, while an increase in tau phosphorylation at this site was also observed in the hippocampus of NP-exposed mice, this difference was not significant compared to the controls (Fig. 4b).

\section{FITC-SiO ${ }_{2}-\mathrm{NPs}$ induced neuroinflammation in the hippocampus}

Neuroinflammation is a crucial pathological hallmark and mediator of neurodegenerative disease. The key players in this process are the activated microglia [24], which proliferate and express/secrete increased levels of pro-inflammatory cytokines, such as interleukin (IL)- $1 \beta$ and IL-6, as well as other inflammatory mediators, such as cyclooxygenase 2 (COX-2) [25]. After exposure to NP, the number of microglia, stained with Iba-1 antibody, was significantly increased in the hippocampus compared to the controls (CA1: $102.10 \%, p=0.0186$; CA3: $56.33 \%, \mathrm{p}=0.0186$; DG: $54.22 \%, p=0.0065$; Fig. 4c). There was also an increase in cell body area in the $\operatorname{mPFC}(51.11 \%, p<0.0001)$ and the hippocampus (CA1: $58.49 \%, \quad \mathrm{p}<0.0001 ; \quad \mathrm{CA} 3: 43.36 \%, \quad p=0.0017 ; \quad \mathrm{DG}$ : $40.23 \%, p=0.0004$ ) of NP group mice (Fig. 4c). These results indicated that NP induced microglial activation in these regions of the brain. Consistent with these data, we also observed a significant increase in the mRNA expression of IL-1 $\beta$ (60.69\%, $p=0.0375$; Fig. $4 \mathrm{~d})$ and IL-6 (41.71\%, $p=0.0441$; Fig. $4 \mathrm{~d})$, and in the protein level of COX-2 (19.21\%, $p=0.0442$; Fig. 4e) in the hippocampus of NP-exposed mice compared to controls. Microglial activation and the upregulation of pro-inflammatory markers in the hippocampus suggest that NP exposure induced neuroinflammation in this region of the brain.

\section{FITC-SiO ${ }_{2}$-NPs activated MAPKs}

MAPKs respond to extracellular stimulation, and MAPK activation has been shown to be directly involved in tau phosphorylation and inflammation in the brain [26]. Thus, we evaluated the roles of three MAPKs, ERK, JNK, and p38, in the observed NP-induced changes in the brain. As shown in Fig. 5, ERK phosphorylation was significantly increased in both the frontal cortex $(44.48 \%, p=0.0051)$ and the hippocampus $(53.66 \%, p=0.0046)$ after NP exposure compared to the controls, while JNK phosphorylation was significantly increased primarily in the frontal cortex $(41.50 \%, p=0.0043)$. These results indicate that ERK and JNK signaling likely played a role in the NP-induced cellular changes in the brain. However, we did not observe any differences in p38 phosphorylation between the NP- and PBS-treated mice nor in the expression of cAMP responsive element binding protein (CREB), which is a downstream pro-survival signaling factor [27] (Additional file 1: Figure S2).

\section{FITC-SiO ${ }_{2}-\mathrm{NPs}$ impaired synaptic exocytosis in the frontal cortex and in primary neuronal cell cultures}

Exocytosis and endocytosis are essential processes in synapse firing, allowing communication to occur between neurons. Using an FM dye assay to analyze the synaptosome fractions isolated from the frontal cortex, we found that exocytosis was significantly impaired in the NP-exposed mice compared to the controls $(-6.76 \%, p=0.0092$; Fig. 6a). However, neither endocytosis nor exocytosis was affected in the hippocampus (Fig. 6a).

We also evaluated endocytosis and exocytosis in primary cultures of cortical neurons. Notably, while the 


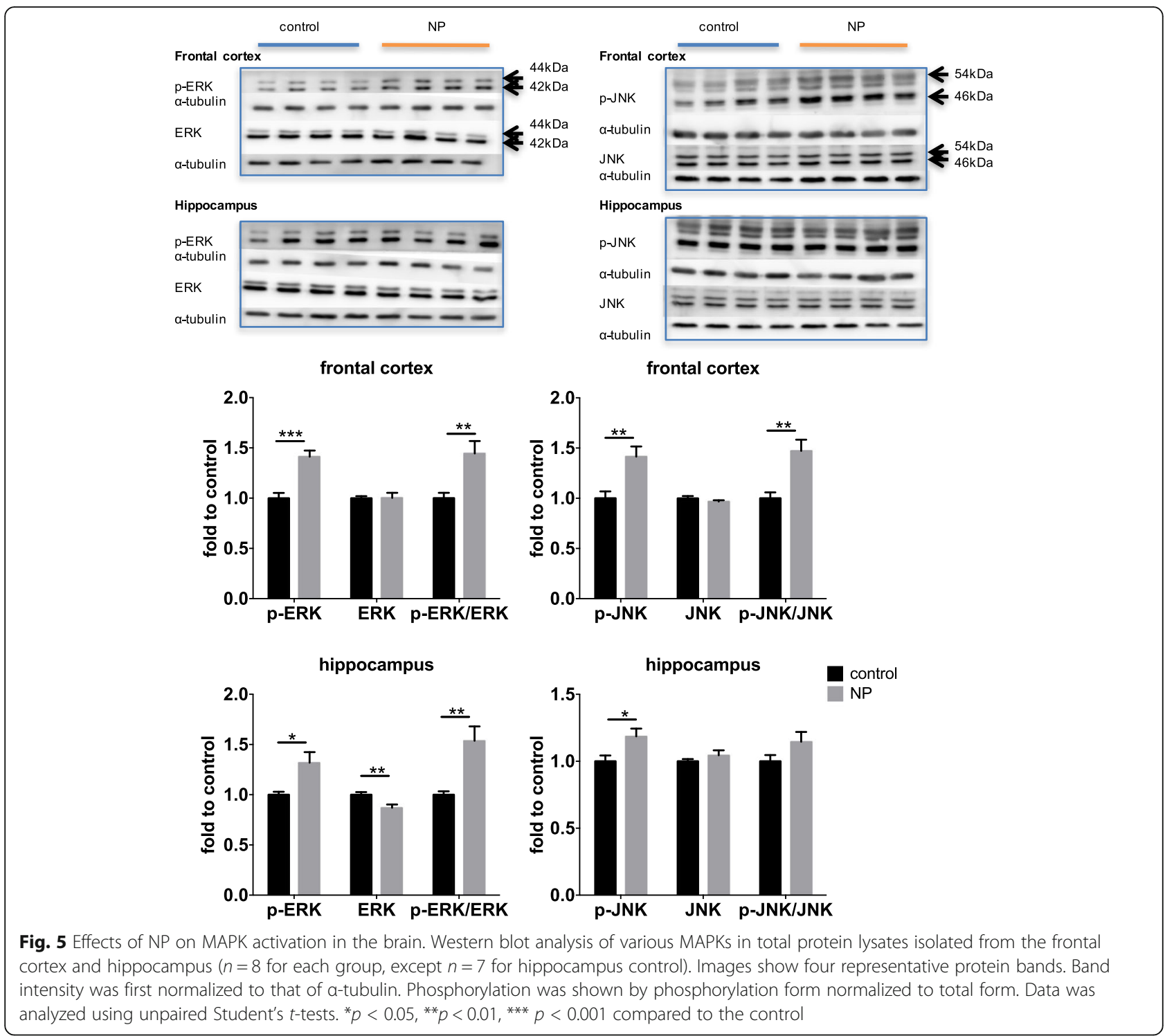

control group neurons efficiently loaded and released the FM 4-64 dye during endocytosis and exocytosis, respectively, these processes were impaired in the NP-exposed neurons (endocytosis: $-19.44 \%, p<0.05$; exocytosis: $72.33 \%, p<0.0001$; Fig. 6b). As endocytosis and exocytosis have also been linked to MAPKs function, we investigated the effects of blocking ERK and JNK activation on these synaptic vesicle transport processes. For this analysis, ERK inhibitor U0126 or JNK inhibitor II (JNKi, SP600125) was used to treat the primary cultures of cortical neurons $1 \mathrm{~h}$ prior to the $48 \mathrm{~h}$ NP exposure. While inhibition of JNK activation did not significantly alter the NP-mediated effects on endocytosis and exocytosis, inhibition of ERK activation significantly blocked the effects of NP exposure on exocytosis in the inhibitor-treated neurons compared to cells treated with NP alone $(-30.44 \%, p<0.001$ vs NP neurons; Fig. 6b). These results indicate that ERK activation may play a significant role in NP-induced synaptotoxicity.

\section{FITC-SiO 2 -NPs altered the expression of important synapse structural proteins}

Synapsin I and synaptophysin are presynaptic proteins with different, but essential functions. We observed a decrease in synapsin I protein expression and an increase in synaptophysin protein expression in both the primary cortical neuron cultures as measured by immunofluorescence shown in Fig. $6 \mathrm{c}$ (synapsin I: $-26.04 \%, p=0.0002$; synaptophysin: $32.32 \%$, $p=0.0009$ ) and by Western-blot shown in Additional file 1: Figure S3 (synapsin I: $-14.18 \%, p=0.0348$; synaptophysin: $41.95 \%, p=0.0096)$, and the synaptosome fraction of the frontal cortex in NP-exposed mice (synapsin I: - 
a

FM 1-43 dye flurorescent intensity

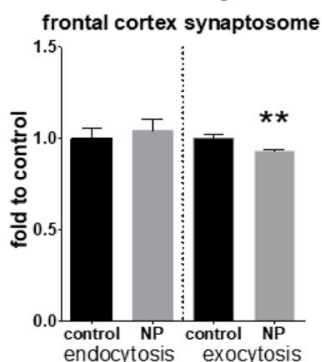

b

$\mathrm{NP}(\mu \mathrm{g} / \mathrm{ml}) \quad 0$

JNKi $(\mu \mathrm{M})$

U0126 ( $\mu \mathrm{M})$
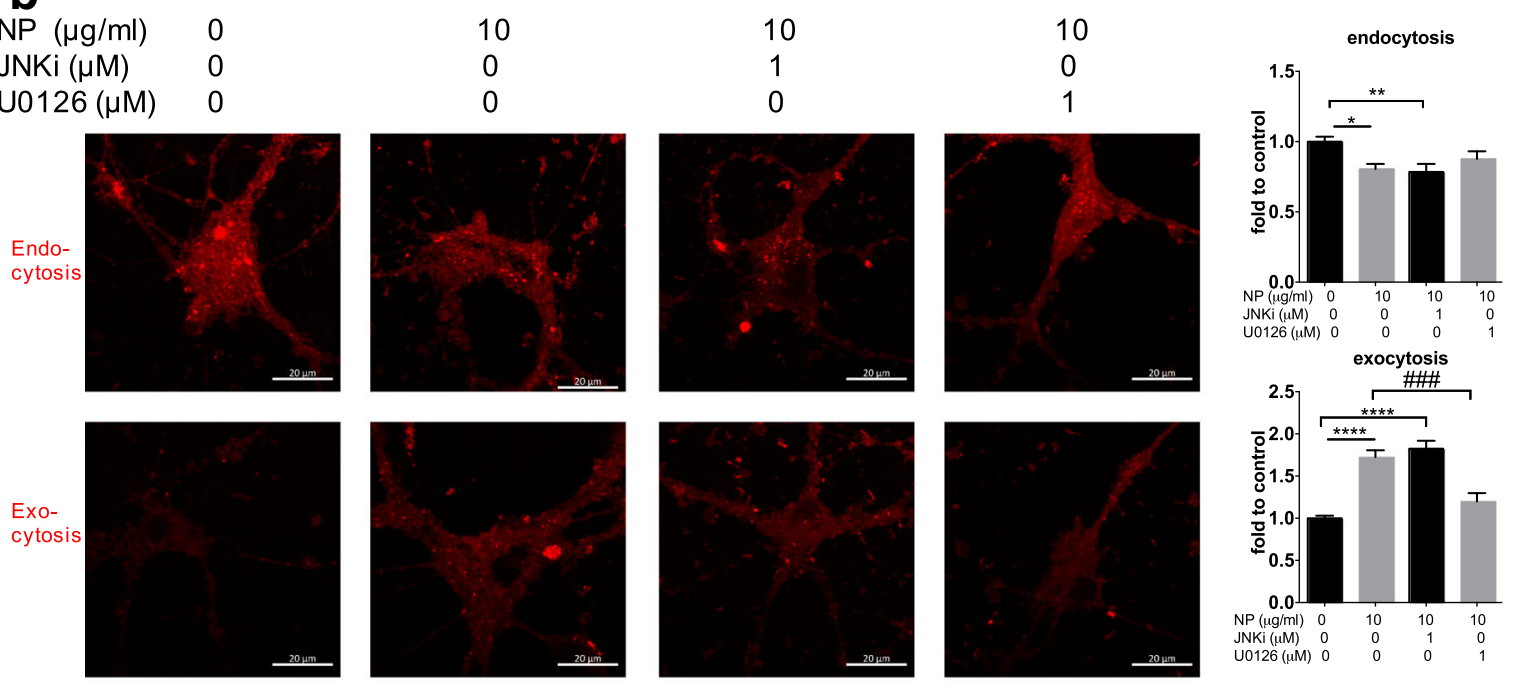

C
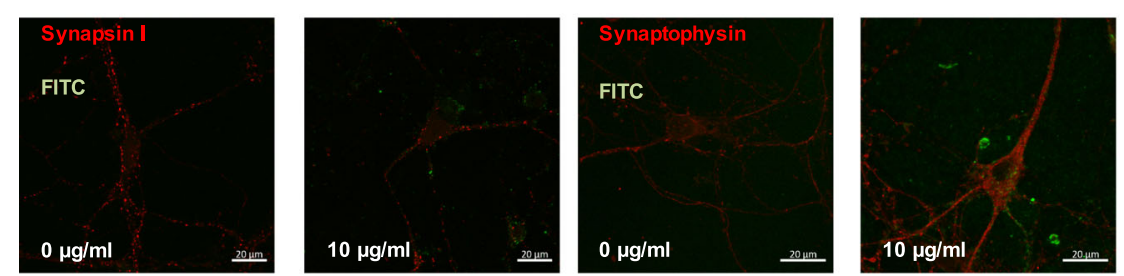

d
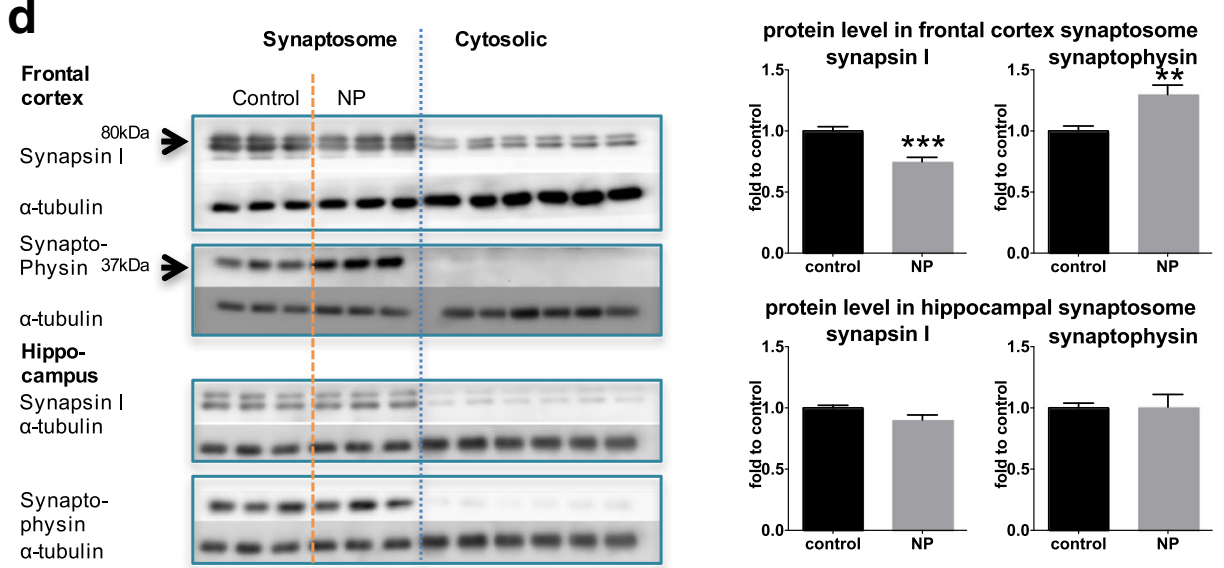

protein level in hippocampal synaptosome
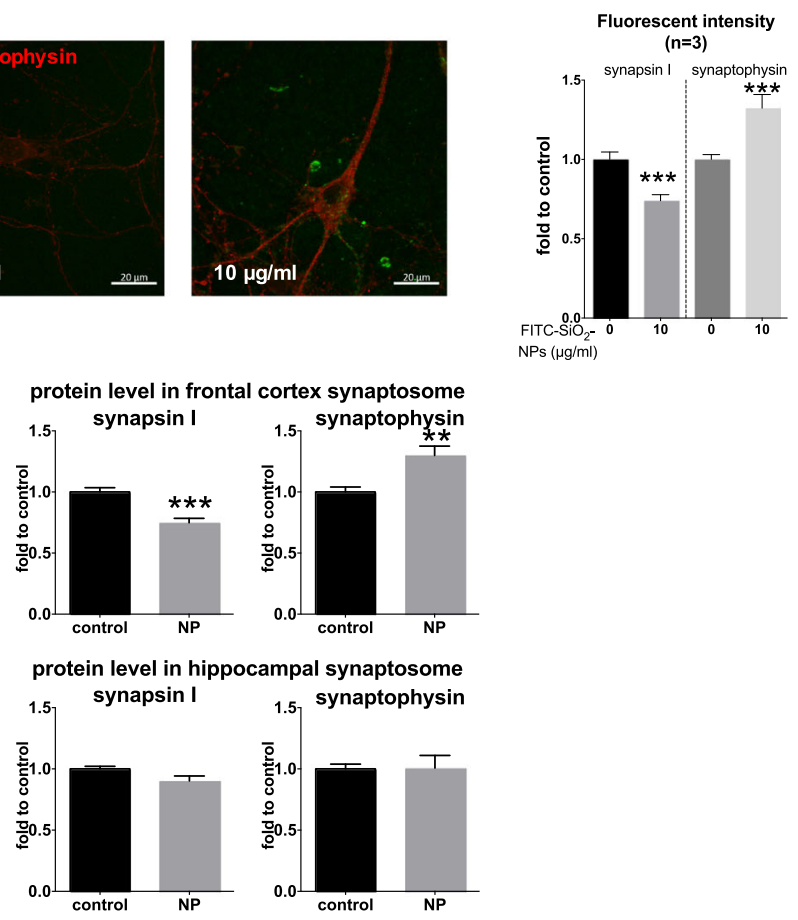

Fig. 6 (See legend on next page.) 


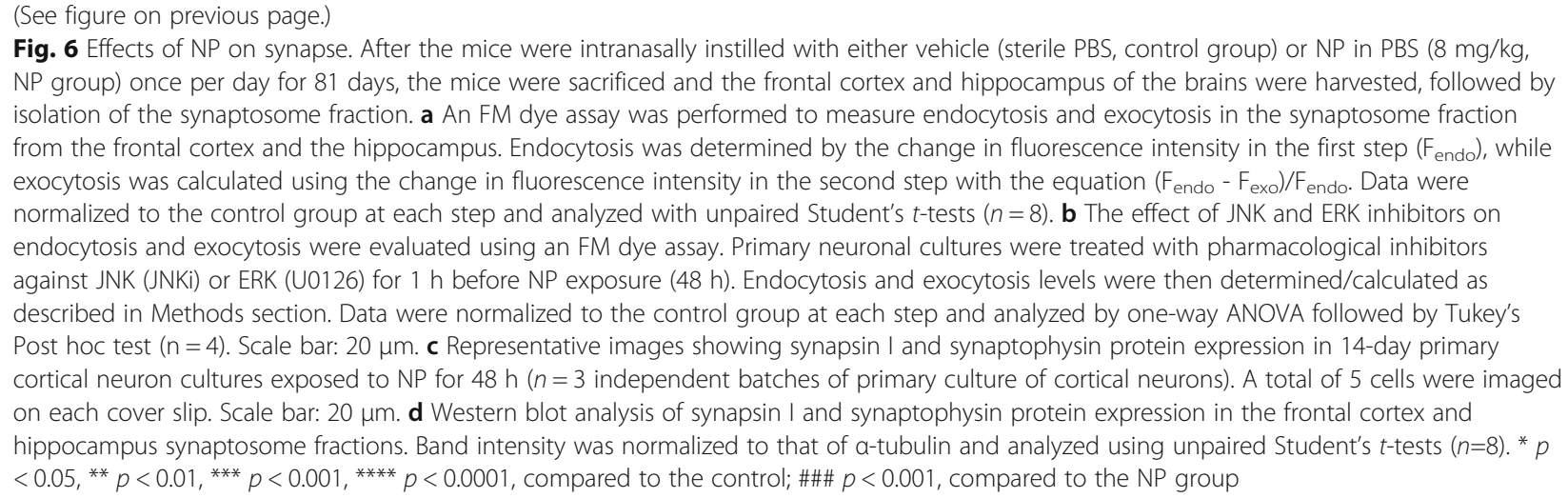

$25.18 \%, \mathrm{p}=0.0002$; synaptophysin: $29.90 \%, p=0.0038$; Fig. 6d) compared to their respective controls. Thus, NP exposure appeared to alter synaptic structure.

\section{Discussion}

Epidemiological and experimental studies have indicated that exposure to nano-scaled particles, varying from fine or ultrafine PM in airborne pollution to artificially engineered NP with different chemical compositions, may induce both cognitive impairment and/or mood dysfunction [28]. $\mathrm{SiO}_{2}$-NPs are a significant component of airborne PM, and exposure has been shown to cause a variety of health issues. Notably, amorphous silica is regarded as less toxic compared to crystalline silica, a well-known carcinogen [29]. However, the long-term effects of respiratory exposure to amorphous $\mathrm{SiO}_{2}-\mathrm{NPs}$ are not fully understood, particularly with regards to neurodegeneration. In the present study, we evaluated the role of amorphous $\mathrm{SiO}_{2}$-NPs in the development of neurodegeneration-like changes in brain pathology and behavior after intranasal instillation in young adult mice.

While this study is not the first to investigate the effects of nanoparticles on behavior, our investigation differs in three major aspects: age of the subjects, exposure time points, and the behavioral tests utilized. Notably, most of the existing studies investigating the effects of nanoparticles have been performed in young subjects, such as children [30,31] or juvenile rodents (4 weeks old) [32-35], or in the elderly [36, 37], while the supporting evidence in adults is limited [38]. Thus, we focused on the impact of $\mathrm{SiO}_{2}$-NPs on young adult mice, whose well-developed brains should, in theory, be more resistant to stimulation [39]. However, our results demonstrate that NP could also affect this age group.

Furthermore, many of the previous studies have primarily focused on the short-term acute effects of $\mathrm{SiO}_{2}$-NPs. Indeed, intranasal instillation of $\mathrm{SiO}_{2}-\mathrm{NPs}$ once per day for 7 days was shown to induce a mild increase in the mRNA expression of pro-inflammatory cytokines in the hippocampus of rats [40]. Further studies investigating nanoparticles also indicated that the effect of short term exposure on behavior may be limited $[41,42]$. Notably, one long-term study showed that exposing mice to PM2.5 for 10 months leads to profound behavioral changes in mood and cognition [32]. Therefore, in order to focus on the earliest, but significant, behavioral changes, we focused on the 1 and 2 month exposure time points in the present study.

To get a comprehensive understanding of the effects of $\mathrm{SiO}_{2}$-NPs exposure on behavior, we designed a battery of tests to evaluate multiple behavioral domains, including motor function, mood, and cognition. At least two tests were included for each, and although the tests for the same domain differ in sensitivity, motivation, and even behavior subcategory, the results revealed the same changes or at least the same trend in each domain. In fact, according to our results, social interaction activity was the most sensitive to NP treatment, being significantly reduced at both time points. At the 2 month time point, we also observed increased anxiety as well as impairments in memory and spatial learning. Therefore, it would appear that $\mathrm{SiO}_{2}$-NPs exposure causes significant changes in multiple behavioral domains, with most of these behaviors being affected after 2 months of exposure. Moreover, no significant differences were observed in the motor function tests, thus excluding any potential physical biases in the other tests.

One of the major concerns when studying behavior is the order of tests and the amount of rest between tests. In the present study, the tests were arranged under the principle of minimum interference and maximum habituation to decrease the adverse influence of one test on another. The mice were only exposed to one test per day, except for the elevated plus maze test, which followed the open field test as suggested in the literature [43]. Tests without externally driven forces, such as the open field test, elevated plus maze test, novel object recognition test, and social interaction test, were performed 
first. The interference could be further minimized by gradually increasing the level of stimulation involved in the tests, from none (open field test) to a novel object (novel object recognition test) and then to a novel mouse (social interaction test). As the motivation and stimulation levels in the rotarod test, Morris water maze test, and the despair tests gradually increase, these tests were performed later in the testing period and in this order. Interestingly, some test experiences may improve the stability and reliability of the outcomes of the following tests [43, 44]. This is largely because the initial experience could increase habituation. Sufficient habituation is of vital importance with regards to behavioral test performance, particularly for mice, which are highly sensitive to external stimulation. For example, the arena used for the open field test was also used for the later novel object recognition test and social interaction test, which could allow the animal to become familiar with their surroundings and focus on the tests.

In addition to behavior, we also evaluated the neuropathological changes induced by NP exposure. It was essential to first evaluate if the NP were translocated to the brain after intranasal instillation. To detect NP deposition in sections of the brain, which is a well-known autofluorescent tissue, we used an anti-FITC antibody to magnify the fluorescent intensity of the NP. This method allowed us to study the regional localization of the NP. Indeed, we detected the NP in sub-regions of the frontal cortex (mPFC) and the hippocampus (CA1 and CA3). These data are supported by a previous report showing similar deposition in the brains of rats exposed to ${ }^{125}$ I-labeled $\mathrm{SiO}_{2}$-NPs for 7 days [40]. Furthermore, this deposition pattern also correlated with the results of our behavioral tests as these two regions of the brain are critical for mood and cognition.

Surprisingly, we did not observe NP deposition in the DG, whose function is closely related to mood and cognition $[45,46]$. However, neuroinflammation was observed in the DG as well as the CA1 and CA3 in the hippocampus, as indicated by the activation of microglia, and increased expression of IL- $1 \beta$ and IL- 6 mRNA, and COX-2 protein in this region of the brain in NP-treated mice compared to the controls. These findings indicate that NP may affect the DG and other sub-regions of the brain by creating a pro-inflammatory environment.

Detecting pro-inflammatory changes in the brain after NP exposure was not altogether surprising as neuroinflammation was previously shown to be induced in both $\mathrm{SiO}_{2}$-NPs-exposed immortalized microglial cell cultures [47] and in the hippocampus after 7 days of $\mathrm{SiO}_{2}-\mathrm{NPs}$ exposure via intranasal instillation [40]. This type of NP-mediated inflammatory response is also strikingly similar to that observed in neurodegenerative diseases [3], particularly with regards to the downstream signaling molecules. Indeed, IL-1 $\beta$ is neurotoxic [48] and exacerbates neurodegenerative pathology and behaviors in multiple disease models, including AD [49], PD [50], poly I:C-induced neurodegeneration [51], and multiple sclerosis [52]. Further, the plasma levels of IL-6 have also been implicated as a biomarker for FTD [53] and AD [54]. COX-2 is known to be toxic to neurons and synapses [55] and has also been correlated with anxiety-like behavior [56] and cognitive impairment [57] in models of AD and PD [58]. Taken together, it is likely that neuroinflammation in the hippocampus contributes to the observed NP-induced behavioral changes.

Although neuroinflammation was mainly observed in the hippocampus, we also detected other neurodegeneration-like pathological changes including morphological changes in Nissl staining and increased phosphorylation of tau in the frontal cortex. Such a differentiated respond in these two regions of the brain may be due to their different neuroanatomy and intrinsic cellular responses to different stimuli. Similar differences were also found in response to other nanoparticles [59-61], toxins [62], and stress [63]. Noteworthy, NP exposure also induced synaptic changes in the frontal cortex. Synapses, especially the presynaptic terminals, are crucial for nanoparticles uptake into neurons because they are a location of active synaptic vesicle recycling via constant endocytosis and exocytosis [64]. Our results show that exocytosis was profoundly affected in both the synaptosome fraction isolated from the brains of NPs-exposed mice and in primary neuronal cultures exposed to NPs. Synaptic endocytosis and exocytosis are largely orchestrated by structural synaptic proteins and other subcellular structures, such as the cytoskeleton [64]. Therefore, functional changes in the synapses after NP treatment are often accompanied by structural changes. Indeed, we found that in addition to disrupted exocytosis, NP treatment also decreased synapsin I protein expression. Synapsin I is the primary linkage between the synaptic vesicles and the cytoskeleton. Decrease in synapsin I may lead to disrupted synaptic vesicle recycling [65]. Furthermore, expression of synaptophysin, an important structural protein in the synaptic vesicle membrane [65], increased after NP treatment in the synaptosome fractions as well as in cultured neurons. This increase is likely due to the accumulation of synaptic vesicles in the synaptic terminals. These data are similar to those observed for silver nanoparticles, which after being deposited in neuronal cell bodies, induced ultrastructural changes in the synapse and altered synapsin I and synaptophysin protein levels in the hippocampus and cortex of rats [66]. These findings suggest that presynaptic terminals are susceptible to NPs. 
Collectively, this study implicates $\mathrm{SiO}_{2}$-NPs in the development of neurodegeneration-like changes in behavior, neuropathology, and synapse. However, it is also important to consider the mechanism underlying these changes. In previous studies, $\mathrm{SiO}_{2}-\mathrm{NPs}$ have been shown to activate MAPKs in multiple cell types and elicit a variety of responses. For example, $\mathrm{SiO}_{2}$-NPs treatment induced proliferation in human adipose tissue-derived stem cells via activation of ERK [67]. In bronchial epithelial cells [68] and macrophages [69], $\mathrm{SiO}_{2}-\mathrm{NPs}$ also activated ERK, leading to inflammation and oxidative stress. In neurodegenerative disease, chronic activation of ERK has been found to mediate neurotoxicity [70], neuroinflammation [71], and tau hyperphosphorylation [4]. In the current study, we also observed prolonged activation of ERK and JNK accompanied by neuropathology and neuroinflammation following NP exposure, suggesting that activation of these MAPKs may play a key role in the adverse effects of NP in the brain. Furthermore, ERK activation also appears to regulate presynaptic terminal function and inhibits exocytosis in primary cortical neuron cultures [72]. To study these effects in our NP-exposed primary neuronal cultures, we pre-treated the neurons with U0126 or SP600125. U0126 is a potent and selective inhibitor of MEK1 and MEK2 kinases [73] and is one of the most widely used inhibitors of the Ras/Raf/MEK/ERK signaling pathway [74], while SP600125 is a reversible, ATP-competitive inhibitor of JNK1/2 [75]. Our data show that pharmacological inhibition of ERK activation prevented the NP-mediated decrease in exocytosis in the cultured neurons. These results strongly agree with previous findings and highlight a distinct regulatory role for ERK in the synapse. Taken together, it appears that the effects of $\mathrm{SiO}_{2}$-NPs on behavior, neuropathology, and synaptic function are, at least in part, regulated by ERK activation.

\section{Conclusions}

Taken together, our study provides compelling evidence demonstrating that long-term NP exposure affects cognitive functions and mood, induces neurodegeneration-like changes in the brain, and alters synapse. These changes also appear to be related to the regulation of MAPKs activation. To our knowledge, this longitudinal study is the first published indication that 2 months of intranasal exposure to NP affects multiple aspects in the brains of young adult mice, ultimately leading to neurodegeneration. Therefore, $\mathrm{SiO}_{2}$-NPs, which are both environmental-enriched and broadly applied, may be a risk factor for neurodegenerative diseases even in young adult individuals with a higher resistance to foreign stimulus.

\section{Methods}

\section{Synthesis and characterization of NPs}

In the present study, FITC-SiO 2 -NPs were used to represent amorphous $\mathrm{SiO}_{2}$-NPs. The $\mathrm{FITC}-\mathrm{SiO}_{2}-\mathrm{NPs}$ were synthesized with the Stöber method [76, 77]. Using this method, the FITC was linked to the $\mathrm{SiO}_{2}-\mathrm{NPs}$ via an interaction between the modified amine group of the FITC and the silane group on the $\mathrm{SiO}_{2}-\mathrm{NPs}$, thereby trapping the FITC in the silica layer [78]. The full synthesis procedure utilized for the construction of the FITC-SiO ${ }_{2}-\mathrm{NPs}$ can be found in the Additional file 1. All of the chemicals used in this study were purchased from Sigma-Aldrich (St. Louis, Missouri, USA), unless otherwise noted.

The morphology of the NP was characterized with TEM (Tecnai G2 20 S-TWIN TEM, FEI, Oregon, US). The TEM images were then used to determine the average diameter of the FITC-SiO ${ }_{2}$-NPs using Image J software (http://rsb.info.nih.gov/ij/). The hydrodynamic diameter and zeta potential of the NP in PBS (the vehicle used to administer the NP to the mice), neuronal culture medium (1.6\% fetal bovine serum), and water were measured by DLS using a low volume disposable sizing cuvette (ZEN0112) on a Zetasizer Nano (Malvern, Worcestershire, UK). Before each measurement, the NP were sonicated with a Fisher Sonic Dismembrator (Model 300, Markham, Ontario, CA) at 35\% amplification for $15 \mathrm{~min}$. The sonicated samples were then diluted to $1 \mathrm{mg} / \mathrm{ml}$ with distilled water and filtered through a $0.22 \mu \mathrm{m}$ nylon membrane filter. The z-averaged hydrodynamic mean diameter, zeta potential, and polydispersity of the samples were calculated using software provided by Malvern (Worcestershire, UK).

\section{Animals, treatment, and tissue sampling}

Male C57BL/6 N mice (3 months old) were group-housed in cages with regular rodent chow and tap water provided ad libitum in the clean area of the Laboratory Animal Unit at The University of Hong Kong. The light cycle of the animals was from 7 am to $7 \mathrm{pm}$, and all of the experimental handling, such as administration and behavioral tests, took place during this light cycle. To minimize NP aggregation in the aqueous medium, the NP were sonicated (Fisher Sonic Dismembrator, Model 300) at 35\% amplification for $15 \mathrm{~min}$ right before intranasal administration. To minimize the disturbance from handling during administration to the results of behavioral tests, the administration was undertaken after behavioral test by the same operator at the same time of the day in the same room. The protocols used in this study were approved by the Committee on the use of Live Animals in Teaching and Research, The University of Hong Kong. In total, 40 animals were randomly assigned to two groups: PBS-treated (control) and NP-treated. 
The recommended maximum level of amorphous silica in the workplace varies from $2 \mathrm{mg} / \mathrm{m}^{3}$ in New Jersey [79] to the $10 \mathrm{mg} / \mathrm{m}^{3}$ limit set by the American Conference of Governmental Industrial Hygienists, an organization which sets workplace chemical exposure limitations [80]. Therefore, depending on the job location, a $60 \mathrm{~kg}$ worker present in the workplace for $8 \mathrm{~h}$ without proper protection will breathe in approximately $6.7 \mathrm{~m}^{3}$ of air containing 13.3 to $66.7 \mathrm{mg}$ of amorphous silica, resulting in an approximate dose of 0.22 to $1.1 \mathrm{mg} / \mathrm{kg} /$ day. To reflect these levels, the dosage should be 2.7 to $13.53 \mathrm{mg} / \mathrm{kg}$ in mice based on body surface area [81]. In the present study, the dosage for mice was set at the median value, $8 \mathrm{mg} / \mathrm{kg}$.

Thus, mice in the control and NP groups were administered $20 \mu \mathrm{l}$ of vehicle (PBS) or $\mathrm{FITC}^{-\mathrm{SiO}_{2}-\mathrm{NPs}}$ $(8 \mathrm{mg} / \mathrm{kg}, 10 \mathrm{mg} / \mathrm{ml}$ in $\mathrm{PBS})$, respectively, through bilateral intranasal instillation. The intranasal instillation was done with a $20 \mu \mathrm{l}$ pipette with an extended-end tip. The fully conscious mouse was handled with its head up, and the end of the pipette tip was placed near the nostril. Each instillation was separated into four smaller aliquots to ensure minimal leakage to the mouth. The treatment duration was 81 days.

After perfusion with normal saline, the brain was dissected and the frontal cortex and hippocampus were isolated [82]. For synaptosome extraction, the tissue samples were extracted with Syn-PER reagent (Thermo Fisher, Waltham, Massachusetts, USA) supplemented with protease and phosphatase inhibitors according to the manufacturer's protocol. For total lysate extraction, the tissue samples were homogenized with RIPA buffer (Cell Signaling Technology, Danvers, Massachusetts, USA) supplemented with protease and phosphatase inhibitors. RNA was extracted from the tissue samples with RNAiso Plus reagent (Takara, Kusatsu, Shiga, Japan) and cDNA was synthesized using PrimeScript RT Master Mix (Takara, Kusatsu, Shiga, Japan) as previously described [83]. To prepare frozen embedded tissue, half of the perfused brain was post-fixed in $4 \%$ paraformaldehyde (PFA) for $72 \mathrm{~h}$ and then soaked in $30 \%$ sucrose in phosphate buffer $(\mathrm{PB})$ until saturated. The tissues were then embedded in tissue freezing medium (Leica, Wetzlar, Germany) and sectioned with a cryostat (Leica, Wetzlar, Germany). The sections were stored in PBS at $4{ }^{\circ} \mathrm{C}$ until free-floating staining was performed.

\section{Behavioral tests}

\section{Grouping and behavioral test arrangement}

During treatment (at 1 month and 2 months), mice were tested with a battery of behavioral tests, as shown in Fig. 2a. The order of these behavioral tests was from the least to the most stressful to the animals. Notably, some behavioral tests can be repeated, such as the novel object recognition test when different pairs of objects are used, while others, such as the Morris water maze test, cannot be repeated because the outcome may be affected by the previous experience. For this reason, the control and NP-treated mice tested at 1 month ( $n=8$ per group) were not tested again for some experiments, while a second set of control and NP-treated mice ( $n=12$ per group) were tested only at the 2 month time point.

To study cognition in the treated mice, we used the novel object recognition test with a $24 \mathrm{~h}$ inter-session interval to study their memory and the Morris water maze test to study their spatial learning and memory. Furthermore, since the NP were administered via intranasal instillation, it is possible that cardiopulmonary function could be affected, making it essential to evaluate any aberrant effects on motor function. We used the open field test and the accelerating rotarod test to study the locomotor and motor functions after NPs exposure, respectively. Moreover, it was also important to understand any psychological changes associated with treatment. Social interaction activity is an important aspect affected in anxiety and depression, both of which are commonly observed during neurodegeneration [23]. To elucidate any anxiety-related symptoms, we use the elevated plus maze test, the golden standard for measuring anxiety [43]. In addition, central area duration during the open field test can also serve as an indicator for anxiety [84]. The forced swimming test and tail suspension test, also known as 'despair tests', were used to test for depression, which is measured in relation to the immobility time during the tests.

The protocols utilized for the open field test [84], elevated plus maze test [43], novel object recognition test [44], social interaction test [83], accelerating rotarod test [83], Morris water maze test [85], forced swimming test [86], and tail suspension test [87] were similar to those reported in the literature. A detailed protocol for each is provided in the Additional file 1.

\section{Primary culture of cortical neurons}

Primary cultures of cortical neurons were prepared from embryonic day 18 (E18) Sprague-Dawley rats similar to a previous report [88]. A detailed protocol is provided in the Additional file 1 . This protocol was approved by the Committee on the use of Live Animals in Teaching and Research at The University of Hong Kong.

After 12 days in culture, the cortical neurons were exposed to $2 \mathrm{ml}$ of medium (2:1 ratio of complete Neurobasal medium and Minimum Essential medium supplemented with $1.6 \%$ fetal bovine serum) with PBS or $\mathrm{NP}$ at a concentration of $10 \mu \mathrm{g} / \mathrm{ml}$. Notably, the NP were sonicated immediately prior to use to avoid aggregation, as described above. The cells were incubated in a $37{ }^{\circ} \mathrm{C}$ inbubator with $5 \% \mathrm{CO}_{2}$ for $48 \mathrm{~h}$ and then analyzed 
using the FM dye assay or immunocytochemical staining. To further study the role of ERK and JNK activation in the NP-mediated changes, neurons were pre-treated with $1 \mu \mathrm{M}$ of either U0126 or JNKi $1 \mathrm{~h}$ before exposure to NP for $48 \mathrm{~h}$. These treatments were performed according to the manufacturer's instructions for U0126 as well as previous reports for JNKi [89]. After treatment with NPs, the neuronal cultures were analyzed using the FM dye assay.

\section{FM dye assay}

FM dyes label the lipid membrane and have, therefore, been used to monitor endocytosis and exocytosis in synaptic vesicles in both cell culture and synaptosomal fractions of brain tissues. With this assay, the gain and loss of fluorescence demonstrates the efficiency of endocytosis and exocytosis in the synapse, respectively.

The freshly prepared synaptosome fractions were quantified and diluted in Hanks' balanced salt solution (HBSS, Gibco, US), and then incubated with $100 \mu \mathrm{M}$ of FM1-43 dye (Thermo Fisher, Waltham, Massachusetts, USA) and $30 \mathrm{mM} \mathrm{KCl}$ at room temperature for $15 \mathrm{~min}$ to activate endocytosis and to induce dye uptake. Then, the mixture was centrifuged at $15000 \times \mathrm{g}$ for $5 \mathrm{~min}$ at $4{ }^{\circ} \mathrm{C}$ and washed with HBSS twice. Each pellet was resuspended in $350 \mu \mathrm{l}$ of HBSS, and $100 \mu \mathrm{l}$ of the suspension was added to a 96-well plate (in triplicate) for analysis on a plate-reader (Perkin Elmer, Waltham, Massachusetts, USA) at an excitation/emission of 530/600 nm. This was designated the endocytosis reading $\left(\mathrm{F}_{\text {endo }}\right)$. Upon the finishing this analysis, the synaptosome suspensions in the wells were collected and incubated with $30 \mathrm{mM} \mathrm{KCl}$ for $15 \mathrm{~min}$ at room temperature to activate exocytosis, followed by centrifugation, washing, and resuspension as described above. The fluorescence analysis was designated the exocytosis reading $\left(\mathrm{F}_{\text {exo }}\right)$. Exocytosis function of the sample of synapses was calculated as $\left(\mathrm{F}_{\text {endo }}-\mathrm{F}_{\text {exo }}\right) / \mathrm{F}_{\text {endo }}$. The results of this analysis for both the endocytosis and exocytosis readings are shown as the fold change compared to the control.

The FM dye assay protocol used to analyze the primary neuronal cultures was similar to that previous reported [90]. Briefly, FM4-64FX (LifeTechnologies, Carlsbad, California, USA) fluorescent dye was dissolved in HBSS to achieve a working concentration of $5 \mu \mathrm{M}$. The primary cortical neurons were cultured in 6-well plates on coverslips, two per well. Each well represented one treatment condition. After treatment, the culture medium was removed and the neurons were briefly washed with HBSS, followed by stimulation with $100 \mathrm{mM} \mathrm{KCl}$ for $5 \mathrm{~min}$ to induce endocytosis. After washing with HBSS, the cells were incubated with dye for $30 \mathrm{~min}$ at $37^{\circ} \mathrm{C}$. Then, the cells were washed and one group was fixed with $4 \%$ PFA for $13 \mathrm{~min}$. The remaining neurons were then stimulated with $100 \mathrm{mM}$ potassium chloride for $10 \mathrm{~min}$ to induce exocytosis and then fixed with $4 \%$ PFA after washing. The coverslips were mounted on microscope slides using ProLong ${ }^{\circ}$ Gold antifade mounting medium (LifeTechnologies, Carlsbad, California, USA). Images were taken with a LSM510-meta laser scanning confocal microscope (Carl Zeiss, Oberkochen, Germany) and quantified using ImageJ (http://rsb.info.nih.gov/ij/). The average fluorescence intensity in both the endocytosis $\left(\mathrm{F}_{\text {endo }}\right)$ and exocytosis $\left(\mathrm{F}_{\text {exo }}\right)$ steps were normalized to the control group to show the change in function after NP exposure.

\section{Western blot}

Western-blot analysis was performed as previously reported [91]. The full protocol and antibodies using in this study are listed in the Additional file 1.

\section{Real time-PCR (RT-PCR)}

RT-PCR was performed using SYBR Premix Ex Taq ${ }^{\text {Ti }}$ II kit (Takara, Shiga, Japan) and employing the StepOnePlus Real-Time PCR System (Applied Biosystems, Thermo Fisher Scientific, USA) as previously reported [83]. The primers for IL-1 $\beta$, IL- 6 and GAPDH were the same as in literature [83].

\section{Immunofluorescence, immunohistochemistry, and image analysis}

In the primary cortical neuron cell cultures, the expression of various synaptic proteins was studied using immunocytochemical staining and imaged with an LSM510-meta laser scanning confocal microscope (Carl Zeiss, Oberkochen, Germany) equipped with a $63 \mathrm{X}$ oil objective lens. Five representative neurons were imaged on each coverslip. The fluorescence intensity of the images was quantified with Image J. Alternatively, the brain tissue sections from every 9 sections of the mPFC (bregma $+2.68 \mathrm{~mm}$ to $+1.54 \mathrm{~mm}$ ) and every 15 sections of the hippocampus (bregma $-1.34 \mathrm{~mm}$ to $-2.46 \mathrm{~mm}$ ) were evaluated using immunohistochemistry or immunofluorescence. A total of three non-overlapping fields in the mPFC as well as the CA1, CA3, and DG in the hippocampus were imaged. The detailed protocols and antibodies used in these analyses are shown in the Additional file 1.

Notably, the deposition of NP was studied using immunofluorescent staining of free-floating frozen brain sections from three representative animals per group, followed by confocal microscopy (LSM 700, Carl Zeiss, Oberkochen, Germany). Microglia in the mPFC and hippocampus were labeled with an Iba-1 antibody and imaged by confocal microscopy. Quantification of microglia cells in the area of interest was done with Image J. The detailed protocol for measuring the number and 
area of the stained cells can be found in the Additional file 1. Nissl staining was also performed to reveal granules, known as Nissl bodies, in the neuronal cytoplasm using a previously reported protocol [92]. A Zeiss Axiophot microscope (Carl Zeiss, Thornwood, US) with a 20X objective lens and a SPOT RT3TM camera (SPOT Imaging Solutions, Sterling Heights, US) was used to acquire the images. Scale bar for confocal microscopic images and light field images was added by ZEN (https://www.zei ss.com.cn/microscopy/downloads/zen.html\#inpagetabs-0) and AxioVision (https://www.zeiss.com/microscopy/int/ downloads/axiovision-downloads.html), respectively. Notably, the researcher processing all the images was blinded to the treatment groups to avoid bias.

\section{Statistical analysis}

All data, including that from the behavioral tests, western blot analysis, immunohistochemistry, and in vitro studies, were first examined by the Shapiro-Wilk normality test to test for normal distribution. If the data was normally distributed, a two-tailed Student's $t$-test or one-way ANOVA was used for statistical analysis. If the data were not normally distributed, the Mann-Whitney test was used. To analyze the data obtained for the Morris water maze test training phase or the rotarod test, a repeated-measured ANOVA was conducted with treatment as the within-subject factor and time as the between-subject factor. All statistical analyses were conducted with Prism 6 software (GraphPad Software, La Jolla, California, USA). All results are presented as the means \pm standard error of the mean (s.e.m.). In all cases, a $p$-value less than 0.05 was considered statistically significant.

\section{Additional file}

Additional file 1: (Methods. Effects of FITC-SiO 2 -NPs on behavior in mice (Figure S1.) Effects of FITC-SiO - -NPs on phosphorylations of p38 and CREB and its effectors in total lysates of frontal cortex and hippocampus (Figure S2.) Protein levels of synaptophysin and synapsin I in the primary culture of cortical neurons exposed to NP for $48 \mathrm{~h}$ as analyzed by Western blot (Figure S3.) DOCX 1064 kb)

\section{Abbreviations}

AD: Alzheimer's disease; CA: Cornu ammonis area; COX-2: Cyclooxygenase 2; CREB: CAMP responsive element binding protein; DG: Dentate gyrus; DLS: Dynamic light scattering; ERK: Extracellular signal-regulated kinases; FITC: Fluorescein isothiocyanate; FTD: Frontotemporal dementia; HBSS: Hank's balanced salt solution; IL: Interleukin; JNK: c-Jun N-terminal kinases: MAPKs: Mitogen-activated protein kinases; mPFC: Medial prefrontal cortex; PBS: Phosphate buffered saline; PD: Parkinson's disease; PM: Particulate matter; RT-PCR: Real time-PCR; $\mathrm{SiO}_{2}$-NPs: Silica nanoparticles; TEM: Transmission electron microscopy

\section{Acknowledgements}

We appreciate the generous help from Dr. Cora Sau-Wan Lai for lending us the elevated plus maze and for the technical support.

\section{Funding}

The study is partly supported by the HKU Seed Funding for Basic Science Research (201311159171).

\section{Availability of data and materials}

The datasets supporting the conclusions of this article are included within the article and its additional files.

\section{Authors' contributions}

RY and RCC designed the study. RY, YL, CH, SH, HC, and SL performed the experiments. RY, YL and $\mathrm{CH}$ collected and analyzed the data. RY and RCC wrote the manuscript. $\mathrm{YH}, \mathrm{CHH}, \mathrm{CH}, \mathrm{SH}, \mathrm{HL}$, and RCC provided technical support and conceptual advice. RCC supervised the project. All authors discussed the results and commented on the manuscript. All authors read and approved the final manuscript.

\section{Ethics approval}

The protocols in this study involving animals were approved by the Committee on the use of Live Animals in Teaching and Research at The University of Hong Kong (3680-15) and follow all appropriate ethical guidelines.

\section{Competing interests}

The authors declare that they have no actual or potential competing financial interests.

\section{Publisher's Note}

Springer Nature remains neutral with regard to jurisdictional claims in published maps and institutional affiliations.

\section{Author details}

${ }^{1}$ Laboratory of Neurodegenerative Diseases, School of Biomedical Sciences, LKS Faculty of Medicine, The University of Hong Kong, Pokfulam, Hong Kong, SAR, China. ${ }^{2}$ School of Nursing, Faculty of Health and Social Sciences, The Hong Kong Polytechnic University, Hung Hom, Kowloon, Hong Kong, SAR, China. ${ }^{3}$ State Key Laboratory of Brain and Cognitive Sciences, The University of Hong Kong, Pokfulam, Hong Kong, SAR, China. ${ }^{4}$ Department of Chemistry, Hong Kong Baptist University, Kowloon Tong, Hong Kong, SAR, China. ${ }^{5}$ School of Biomedical Sciences, Rm. L4-49, Laboratory Block, Faculty of Medicine Building, 21 Sassoon Road, Pokfulam, Hong Kong. ${ }^{6}$ Present address: Nanjing Key Laboratory of Pediatrics, Children's Hospital of Nanjing Medical University, Nanjing 210008, China.

Received: 16 January 2018 Accepted: 29 May 2018

Published online: 03 July 2018

References

1. Forman MS, Trojanowski JQ, Lee VM. Neurodegenerative diseases: a decade of discoveries paves the way for therapeutic breakthroughs. Nat Med. 2004; 10(10):1055-63. https://doi.org/10.1038/nm1113. http://www.ncbi.nlm.nih. gov/pubmed/15459709

2. Mayeux R, Stern Y. Epidemiology of Alzheimer disease. Cold Spring Harb Perspect Med. 2012;2(8) https://doi.org/10.1101/cshperspect.a006239. http:// www.ncbi.nlm.nih.gov/pubmed/22908189

3. Block ML, Zecca L, Hong JS. Microglia-mediated neurotoxicity: uncovering the molecular mechanisms. Nat Rev Neurosci. 2007;8(1):57-69. https://doi. org/10.1038/nrn2038. http://www.ncbi.nlm.nih.gov/pubmed/17180163

4. Buee L, Bussiere T, Buee-Scherrer V, Delacourte A, Hof PR. Tau protein isoforms, phosphorylation and role in neurodegenerative disorders. Brain Res Brain Res Rev. 2000;33(1):95-130. http://www.ncbi.nlm.nih.gov/pubmed/ 10967355

5. Lo EH. Degeneration and repair in central nervous system disease. Nat Med. 2010;16(11):1205-9. https://doi.org/10.1038/nm.2226. http://www.ncbi.nlm. nih.gov/pubmed/21052074

6. Li M, Long C, Yang L. Hippocampal-prefrontal circuit and disrupted functional connectivity in psychiatric and neurodegenerative disorders. Biomed Res Int. 2015;2015:810548. https://doi.org/10.1155/2015/810548. http://www.ncbi.nlm.nih.gov/pubmed/25918722

7. Morrison JH, Baxter MG. The ageing cortical synapse: hallmarks and implications for cognitive decline. Nat Rev Neurosci. 2012;13(4):240-50. https://doi.org/10.1038/nrn3200. http://www.ncbi.nlm.nih.gov/pubmed/ 22395804 
8. Shiba Y, Santangelo AM, Roberts AC. Beyond the medial regions of prefrontal cortex in the regulation of fear and anxiety. Front Syst Neurosci. 2016;10:12. https://doi.org/10.3389/fnsys.2016.00012. http://www.ncbi.nlm. nih.gov/pubmed/26941618

9. Marttinen M, Kurkinen KM, Soininen $H$, Haapasalo A, Hiltunen M. Synaptic dysfunction and septin protein family members in neurodegenerative diseases. Mol Neurodegener. 2015;10:16. https://doi.org/10.1186/s13024-0150013-z. http://www.ncbi.nlm.nih.gov/pubmed/25888325

10. Cannon JR, Greenamyre JT. The role of environmental exposures in neurodegeneration and neurodegenerative diseases. Toxicol Sci. 2011; 124(2):225-50. https://doi.org/10.1093/toxsci/kfr239. Ihttp://www.ncbi.nlm. nih.gov/pubmed/21914720

11. Brown RC, Lockwood AH, Neurodegenerative Diseases SBR. An overview of environmental risk factors. Environ Health Perspect. 2005;113(9):1250-6. https://doi.org/10.1289/ehp.7567

12. Vance ME, Kuiken T, Vejerano EP, McGinnis SP, Hochella MF Jr, Rejeski D, et al. Nanotechnology in the real world: redeveloping the nanomaterial consumer products inventory. Beilstein J Nanotechnol. 2015;6:1769-80. https://doi.org/10. 3762/bjnano.6.181. http://www.ncbi.n/m.nih.gov/pubmed/26425429

13. Mihranyan A, Ferraz N, Strømme M. Current status and future prospects of nanotechnology in cosmetics. Prog Mater Sci. 2012;57(5):875-910. https:// doi.org/10.1016/j.pmatsci.2011.10.001.

14. Chaudhry Q, Scotter M, Blackburn J, Ross B, Boxall A, Castle L, et al. Applications and implications of nanotechnologies for the food sector. Food Addit Contam Part A Chem Anal Control Expo Risk Assess. 2008;25(3): 241-58. https://doi.org/10.1080/02652030701744538. http://www.ncbi.nlm. nih.gov/pubmed/18311618

15. Douroumis D, Onyesom I, Maniruzzaman M, Mitchell J. Mesoporous silica nanoparticles in nanotechnology. Crit Rev Biotechnol. 2013;33(3):229-45. https://doi.org/10.3109/07388551.2012.685860. http://www.ncbi.nlm.nih.gov/ pubmed/22724458

16. Geysen D, Vandecasteele C, Jaspers M, Wauters G. Comparison of immobilisation of air pollution control residues with cement and with silica. J Hazard Mater. 2004;107(3):131-43. https://doi.org/10.1016/j.jhazmat.2003. 12.001. http://www.ncbi.nlm.nih.gov/pubmed/15072821

17. Brouwer D. Exposure to manufactured nanoparticles in different workplaces. Toxicology. 2010;269(2-3):120-7. https://doi.org/10.1016/j.tox.2009.11.017. http://www.ncbi.nlm.nih.gov/pubmed/19941928

18. Oberdorster G, Sharp Z, Atudorei V, Elder A, Gelein R, Kreyling W, et al. Translocation of inhaled ultrafine particles to the brain. Inhal Toxicol. 2004; 16(6-7):437-45. https://doi.org/10.1080/08958370490439597. http://www. ncbi.nlm.nih.gov/pubmed/15204759

19. Yang X, He C, Li J, Chen H, Ma Q, Sui X, et al. Uptake of silica nanoparticles: neurotoxicity and Alzheimer-like pathology in human SK-N-SH and mouse neuro2a neuroblastoma. Cell Toxicol Lett. 2014;229(1):240-9. https://doi.org/ 10.1016/j.toxlet.2014.05.009. http://www.ncbi.nlm.nih.gov/pubmed/24831964

20. Li X, Liu B, Li XL, Li YX, Sun MZ, Chen DY, et al. SiO2 nanoparticles change colour preference and cause Parkinson's-like behaviour in zebrafish. Sci Rep. 2014:4:3810. https://doi.org/10.1038/srep03810. http://www.ncbi.nlm.nih. gov/pubmed/24448416

21. Delaval M, Boland S, Solhonne B, Nicola MA, Mornet S, Baeza-Squiban A, et al. Acute exposure to silica nanoparticles enhances mortality and increases lung permeability in a mouse model of Pseudomonas aeruginosa pneumonia. Part Fibre Toxicol. 2015;12:1. https://doi.org/10.1186/s12989014-0078-9. http://www.ncbi.nlm.nih.gov/pubmed/25605549

22. Marzaioli V, Aguilar-Pimentel JA, Weichenmeier I, Luxenhofer G, Wiemann $M$, Landsiedel $R$, et al. Surface modifications of silica nanoparticles are crucial for their inert versus proinflammatory and immunomodulatory properties. Int J Nanomedicine. 2014;9:2815-32. https://doi.org/10.2147/IJN S57396. http://www.ncbi.nlm.nih.gov/pubmed/24940059

23. Cummings JL, Diaz C, Levy M, Binetti G, Litvan II. Neuropsychiatric syndromes in neurodegenerative disease: frequency and Signficance. Semin Clin Neuropsychiatry. 1996;1(4):241-7. https://doi.org/10.1053/ SCNP00100241. http://www.ncbi.nlm.nih.gov/pubmed/10320427

24. Ransohoff RM. How neuroinflammation contributes to neurodegeneration. Science. 2016;353(6301):777-83. https://doi.org/10.1126/science.aag2590. http://www.ncbi.nlm.nih.gov/pubmed/27540165

25. Boche D, Perry VH, Nicoll JA. Review: activation patterns of microglia and their identification in the human brain. Neuropathol Appl Neurobiol. 2013; 39(1):3-18. https://doi.org/10.1111/nan.12011. http://www.ncbi.n/m.nih.gov/ pubmed/23252647
26. Zhu X, Lee HG, Raina AK, Perry G, Smith MA. The role of mitogen-activated protein kinase pathways in Alzheimer's disease. Neurosignals. 2002;11(5): 270-81. doi:67426. http://www.ncbi.nlm.nih.gov/pubmed/12566928

27. Kida S, Serita T. Functional roles of CREB as a positive regulator in the formation and enhancement of memory. Brain Res Bull. 2014;105:17-24. https://doi.org/10.1016/j.brainresbull.2014.04.011. http://www.ncbi.nlm.nih. gov/pubmed/24811207

28. Heusinkveld HJ, Wahle T, Campbell A, Westerink RH, Tran L, Johnston H, et al. Neurodegenerative and neurological disorders by small inhaled particles. Neurotoxicology. 2016;56:94-106. https://doi.org/10.1016/j.neuro.2016.07. 007. https://www.ncbi.nlm.nih.gov/pubmed/27448464

29. Borm PJ, Tran L, Donaldson K. The carcinogenic action of crystalline silica: a review of the evidence supporting secondary inflammation-driven genotoxicity as a principal mechanism. Crit Rev Toxicol. 2011;41(9):756-70. https://doi.org/10.3109/10408444.2011.576008. http://www.ncbi.nlm.nih.gov/ pubmed/21923565

30. Calderon-Garciduenas L, Leray E, Heydarpour P, Torres-Jardon R, Reis J. Air pollution, a rising environmental risk factor for cognition, neuroinflammation and neurodegeneration: the clinical impact on children and beyond. Rev Neurol (Paris). 2016;172(1):69-80. https://doi.org/10.1016/j. neurol.2015.10.008. http://www.ncbi.n/m.nih.gov/pubmed/26718591

31. Calderon-Garciduenas L, Mora-Tiscareno A, Franco-Lira M, Zhu H, Lu Z, Solorio $\mathrm{E}$, et al. Decreases in short term memory, $\mathrm{IQ}$, and altered brain metabolic ratios in urban Apolipoprotein epsilon4 children exposed to air pollution. J Alzheimers Dis. 2015;45(3):757-70. https://doi.org/10.3233/JAD142685. http://www.ncbi.nlm.nih.gov/pubmed/25633678

32. Fonken LK, Xu X, Weil ZM, Chen G, Sun Q, Rajagopalan S, et al. Air pollution impairs cognition, provokes depressive-like behaviors and alters hippocampal cytokine expression and morphology. Mol Psychiatry 2011;16 10:987-995, 73; doi: https://doi.org/10.1038/mp.2011.76. http://www.ncbi. nlm.nih.gov/pubmed/21727897.

33. Wu J, Yu C, Tan Y, Hou Z, Li M, Shao F, et al. Effects of prenatal exposure to silver nanoparticles on spatial cognition and hippocampal neurodevelopment in rats. Environ Res. 2015;138:67-73. https://doi.org/10. 1016/j.envres.2015.01.022. http://www.ncbi.nlm.nih.gov/pubmed/25701810

34. Chen YS, Hung YC, Lin LW, Liau I, Hong MY, Huang GS. Size-dependent impairment of cognition in mice caused by the injection of gold nanoparticles. Nanotechnology. 2010;21(48):485102. https://doi.org/10.1088/ 0957-4484/21/48/485102. http://www.ncbi.nlm.nih.gov/pubmed/21051801

35. Han D, Tian Y, Zhang T, Ren G, Yang Z. Nano-zinc oxide damages spatial cognition capability via over-enhanced long-term potentiation in hippocampus of Wistar rats. Int J Nanomedicine. 2011;6:1453-61. https://doi. org/10.2147/IJN.S18507. http://www.ncbi.nlm.nih.gov/pubmed/21796247

36. Ailshire JA, Crimmins EM. Fine particulate matter air pollution and cognitive function among older US adults. Am J Epidemiol. 2014;180(4):359-66. https:// doi.org/10.1093/aje/kwu155. http://www.ncbi.nlm.nih.gov/pubmed/24966214

37. Weuve J, Puett RC, Schwartz J, Yanosky JD, Laden F, Grodstein F. Exposure to particulate air pollution and cognitive decline in older women. Arch Intern Med. 2012;172(3):219-27. https://doi.org/10.1001/archinternmed.2011. 683. http://www.ncbi.nlm.nih.gov/pubmed/22332151

38. Chen JC, Schwartz J. Neurobehavioral effects of ambient air pollution on cognitive performance in US adults. Neurotoxicology. 2009;30(2):231-9. https://doi.org/10.1016/j.neuro.2008.12.011. http://www.ncbi.nlm.nih.gov/ pubmed/19150462

39. Semple BD, Blomgren K, Gimlin K, Ferriero DM, Noble-Haeusslein LJ. Brain development in rodents and humans: identifying benchmarks of maturation and vulnerability to injury across species. Prog Neurobiol. 2013;106-107:116. https://doi.org/10.1016/j.pneurobio.2013.04.001. http://www.ncbi.nlm.nih. gov/pubmed/23583307

40. Wu J, Wang C, Sun J, Xue Y. Neurotoxicity of silica nanoparticles: brain localization and dopaminergic neurons damage pathways. ACS Nano. 2011; 5(6):4476-89. https://doi.org/10.1021/nn103530b. http://www.ncbi.nlm.nih. gov/pubmed/21526751

41. Liu P, Huang Z, Gu N. Exposure to silver nanoparticles does not affect cognitive outcome or hippocampal neurogenesis in adult mice. Ecotoxicol Environ Saf. 2013;87:124-30. https://doi.org/10.1016/j.ecoenv.2012.10.014. http://www.ncbi.nlm.nih.gov/pubmed/2314\6669

42. Xie $Y$, Wang $Y$, Zhang $T$, Ren $G$, Yang Z. Effects of nanoparticle zinc oxide on spatial cognition and synaptic plasticity in mice with depressive-like behaviors. J Biomed Sci. 2012;19:14. https://doi.org/10.1186/1423-0127-1914. http://www.ncbi.nlm.nih.gov/pubmed/22300475 
43. Walf AA, Frye CA. The use of the elevated plus maze as an assay of anxietyrelated behavior in rodents. Nat Protoc. 2007;2(2):322-8. https://doi.org/10. 1038/nprot.2007.44. http://www.ncbi.nlm.nih.gov/pubmed/17406592

44. Leger M, Quiedeville A, Bouet V, Haelewyn B, Boulouard M, Schumann-Bard P, et al. Object recognition test in mice. Nat Protoc. 2013;8(12):2531-7. https://doi.org/10.1038/nprot.2013.155. http://www.ncbi.nlm.nih.gov/ pubmed/24263092

45. Kheirbek MA, Drew LJ, Burghardt NS, Costantini DO, Tannenholz L, Ahmari SE, et al. Differential control of learning and anxiety along the dorsoventral axis of the dentate gyrus. Neuron. 2013;77(5):955-68. https://doi.org/10. 1016/j.neuron.2012.12.038. http://www.ncbi.nlm.nih.gov/pubmed/23473324

46. Tannenholz L, Jimenez JC, Kheirbek MA. Local and regional heterogeneity underlying hippocampal modulation of cognition and mood. Front Behav Neurosci. 2014;8:147. https://doi.org/10.3389/fnbeh.2014.00147. http://www. ncbi.nlm.nih.gov/pubmed/24834033

47. Choi J, Zheng Q, Katz HE, Guilarte TR. Silica-based nanoparticle uptake and cellular response by primary microglia. Environ Health Perspect. 2010;118(5): 589-95. https://doi.org/10.1289/ehp.0901534. http://www.ncbi.nlm.nih.gov/ pubmed/20439179

48. Radesater AC, Johansson S, Oberg C, Luthman J. The vitamin-E analog trolox and the NMDA antagonist MK-801 protect pyramidal neurons in hippocampal slice cultures from IL-1beta-induced neurodegeneration. Neurotox Res. 2003; 5(6):433-42. http://www.ncbi.n/m.nih.gov/pubmed/14715447

49. Kitazawa M, Cheng D, Tsukamoto MR, Koike MA, Wes PD, Vasilevko V, et al Blocking IL-1 signaling rescues cognition, attenuates tau pathology, and restores neuronal beta-catenin pathway function in an Alzheimer's disease model. J Immunol. 2011;187(12):6539-49. https://doi.org/10.4049/jimmunol. 1100620. http://www.ncbi.nlm.nih.gov/pubmed/22095718

50. Pott Godoy MC, Tarelli R, Ferrari CC, Sarchi MI, Pitossi FJ. Central and systemic IL-1 exacerbates neurodegeneration and motor symptoms in a model of Parkinson's disease. Brain. 2008;131(Pt 7):1880-94. https://doi.org/ 10.1093/brain/awn101. http://www.ncbi.nlm.nih.gov/pubmed/18504291

51. Field R, Campion S, Warren C, Murray C, Cunningham C. Systemic challenge with the TLR3 agonist poly I:C induces amplified IFNalpha/beta and IL-1beta responses in the diseased brain and exacerbates chronic neurodegeneration. Brain Behav Immun. 2010;24(6):996-1007. https://doi.org/10.1016/j.bbi.2010.04. 004. http://www.ncbi.nlm.nih.gov/pubmed/20399848

52. Rossi S, Motta C, Studer V, Macchiarulo G, Volpe E, Barbieri F, et al. Interleukin-1 beta causes excitotoxic neurodegeneration and multiple sclerosis disease progression by activating the apoptotic protein p53. Mol Neurodegener. 2014;9:56. https://doi.org/10.1186/1750-1326-9-56. http:// www.ncbi.nlm.nih.gov/pubmed/25495224

53. Gibbons L, Rollinson S, Thompson JC, Robinson A, Davidson YS, Richardson A, et al. Plasma levels of progranulin and interleukin-6 in frontotemporal lobar degeneration. Neurobiol Aging. 2015;36(3):1603 e1-4. https://doi.org/10.1016/j. neurobiolaging.2014.10.023. http://www.ncbi.nlm.nih.gov/pubmed/25435337

54. Rasmuson S, Nasman B, Olsson T. Increased serum levels of dehydroepiandrosterone (DHEA) and interleukin-6 (IL-6) in women with mild to moderate Alzheimer's disease. Int Psychogeriatr. 2011;23(9):1386-92. https://doi.org/10.1017/S1041610211000810. http://www.ncbi.nlm.nih.gov/ pubmed/21729423

55. Sang N, Zhang J, Chen C. COX-2 oxidative metabolite of endocannabinoid 2-AG enhances excitatory glutamatergic synaptic transmission and induces neurotoxicity. J Neurochem. 2007;102(6):1966-77. https://doi.org/10.1111/j. 1471-4159.2007.04668.x. http://www.ncbi.nlm.nih.gov/pubmed/17539917

56. Sil S, Ghosh T. Cox-2 plays a vital role in the impaired anxiety like behavior in colchicine induced rat model of Alzheimer disease. Behav Neurol. 2016; 2016:1501527. https://doi.org/10.1155/2016/1501527. http://www.ncbi.nlm. nih.gov/pubmed/26880859

57. Sil S, Ghosh T. Role of cox-2 mediated neuroinflammation on the neurodegeneration and cognitive impairments in colchicine induced rat model of Alzheimer's disease. J Neuroimmunol. 2016;291:115-24. https:// doi.org/10.1016/j.jneuroim.2015.12.003. http://www.ncbi.nlm.nih.gov/ pubmed/26857505

58. Teismann P, Vila M, Choi DK, Tieu K, Wu DC, Jackson-Lewis V, et al. COX-2 and neurodegeneration in Parkinson's disease. Ann N Y Acad Sci. 2003;991: 272-7. http://www.ncbi.nlm.nih.gov/pubmed/12846993

59. Rahman MF, Wang J, Patterson TA, Saini UT, Robinson BL, Newport GD, et al. Expression of genes related to oxidative stress in the mouse brain after exposure to silver-25 nanoparticles. Toxicol Lett. 2009;187(1):15-21. https://doi.org/10.1016/j. toxlet.2009.01.020. http://www.ncbi.nlm.nih.gov/pubmed/19429238
60. Yuan ZY, Hu YL, Gao JQ. Brain Localization and Neurotoxicity evaluation of Polysorbate 80-modified chitosan nanoparticles in rats. PLoS One. 2015; 10(8):e0134722. https://doi.org/10.1371/journal.pone.0134722. http://www. ncbi.nlm.nih.gov/pubmed/26248340

61. Takahashi Y, Mizuo K, Shinkai Y, Oshio S, Takeda K. Prenatal exposure to titanium dioxide nanoparticles increases dopamine levels in the prefrontal cortex and neostriatum of mice. J Toxicol Sci. 2010;35(5):749-56. http:// www.ncbi.nlm.nih.gov/pubmed/20930469

62. Fowler AK, Thompson J, Chen L, Dagda M, Dertien J, Dossou KS, et al. Differential sensitivity of prefrontal cortex and hippocampus to alcoholinduced toxicity. PLoS One. 2014;9(9):e106945. https://doi.org/10.1371/ journal.pone.0106945. http://www.ncbi.nlm.nih.gov/pubmed/25188266

63. Zlatkovic J, Todorovic N, Boskovic M, Pajovic SB, Demajo M, Filipovic D. Different susceptibility of prefrontal cortex and hippocampus to oxidative stress following chronic social isolation stress. Mol Cell Biochem. 2014; 393(1-2):43-57. https://doi.org/10.1007/s11010-014-2045-z. http://www.ncbi. nlm.nih.gov/pubmed/24671494

64. Fedorovich SV, Alekseenko AV, Waseem TV. Are synapses targets of nanoparticles? Biochem Soc Trans. 2010;38(2):536-8. https://doi.org/10.1042/ BST0380536. http://www.ncbi.nlm.nih.gov/pubmed/20298217

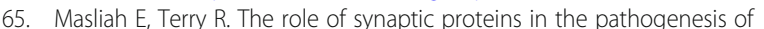
disorders of the central nervous system. Brain Pathol. 1993;3(1):77-85. http:// www.ncbi.n/m.nih.gov/pubmed/8269086

66. Skalska J, Frontczak-Baniewicz M, Struzynska L. Synaptic degeneration in rat brain after prolonged oral exposure to silver nanoparticles. Neurotoxicology. 2015;46:145-54. https://doi.org/10.1016/j.neuro.2014.11.002. http://www. ncbi.nlm.nih.gov/pubmed/25447321

67. Kim KJ, Joe YA, Kim MK, Lee SJ, Ryu YH, Cho DW, et al. Silica nanoparticles increase human adipose tissue-derived stem cell proliferation through ERK1/2 activation. Int J Nanomedicine. 2015;10:2261-72. https://doi.org/10. 2147/IJN.S71925. http://www.ncbi.nlm.nih.gov/pubmed/25848249

68. Eom HJ, Choi J. Oxidative stress of silica nanoparticles in human bronchial epithelial cell, Beas-2B. Toxicol in Vitro. 2009;23(7):1326-32. https://doi.org/ 10.1016/j.tiv.2009.07.010. http://www.ncbi.n/m.nih.gov/pubmed/19602432

69. Park EJ, Park K. Oxidative stress and pro-inflammatory responses induced by silica nanoparticles in vivo and in vitro. Toxicol Lett. 2009;184(1):18-25. https://doi.org/ 10.1016/j.toxlet.2008.10.012. http://www.ncbi.nlm.nih.gov/pubmed/19022359

70. Ghribi O, Prammonjago P, Herman MM, Spaulding NK, Savory J. Abeta(142)-induced JNK and ERK acktivation in rabbit hippocampus is differentially regulated by lithium but is not involved in the phosphorylation of tau. Brain Res Mol Brain Res. 2003;119(2):201-6. http://www.ncbi.nlm.nih.gov/pubmed/ 14625087

71. Sun J, Nan G. The extracellular signal-regulated kinase $1 / 2$ pathway in neurological diseases: a potential therapeutic target (review). Int J Mol Med. 2017;39(6):1338-46. https://doi.org/10.3892/ijmm.2017.2962. http://www. ncbi.nlm.nih.gov/pubmed/28440493

72. Subramanian J, Morozov A. Erk1/2 inhibit synaptic vesicle exocytosis through L-type calcium channels. J Neurosci. 2011;31(12):4755-64. https:// doi.org/10.1523/JNEUROSCI.6594-10.2011. http://www.ncbi.nIm.nih.gov/ pubmed/21430174

73. Duncia JV, Santella JB 3rd, Higley CA, Pitts WJ, Wityak J, Frietze WE, et al. MEK inhibitors: the chemistry and biological activity of U0126, its analogs, and cyclization products. Bioorg Med Chem Lett. 1998;8(20):2839-44. http:// www.ncbi.nlm.nih.gov/pubmed/9873633

74. Ong Q, Guo S, Zhang K, Cui B. U0126 protects cells against oxidative stress independent of its function as a MEK inhibitor. ACS Chem Neurosci. 2015; 6(1):130-7. https://doi.org/10.1021/cn500288n. http://www.ncbi.n/m.nih.gov/ pubmed/25544156

75. Zheng Y, Zhang M, Zhao Y, Chen J, Li B, Cai W. JNK inhibitor SP600125 protects against lipopolysaccharide-induced acute lung injury via upregulation of claudin-4. Exp Ther Med. 2014;8(1):153-8. https://doi.org/10 3892/etm.2014.1684. http://www.ncbi.nlm.nih.gov/pubmed/24944614

76. Rossi LM, Shi L, Quina FH, Rosenzweig Z. Stober synthesis of monodispersed luminescent silica nanoparticles for bioanalytical assays. Langmuir. 2005;21(10): 4277-80. http://www.ncbi.nlm.nih.gov/pubmed/16032835

77. Lin Y-S, Tsai C-P, Huang H-Y, Kuo C-T, Hung Y, Huang D-M, et al. Well-ordered mesoporous silica nanoparticles as cell markers. Chem Mater. 2005;17(18):4570-3.

78. Salqueiriño-Maceira V, Correa-Duarte MA, Spasova M, Liz-Marzán LM, Farle M. Composite silica spheres with magnetic and luminescent functionalities. Adv Funct Mater. 2006:16(4):509-14. https://doi.org/10.1002/adfm. 200500565. https://onlinelibrary.wiley.com/doi/abs/10.1002/adfm.200500565 
79. New Jersey Department of Health and Senior Services: Hazardous substance fact sheet-silica amorphous. Edited by New Jersey Department of Health and Senior Services http://nj.gov/health/eoh/rtkweb/documents/fs/1655.pdf 2003.

80. Wisconsin Department of Natural Resources. Report to the Natural Resources Board: Silica Study 2011. dnr.wi.gov/files/PDF/pubs/am/AM407.pdf.

81. FDA: Guidance for industry-estimating the maximum safe starting dose in initial clinical trials for therapeutics in adult healthy volunteers. https://www. fda.gov/downloads/drugs/guidances/ucm078932.pdf 2005.

82. Chiu K, Lau WM, Lau HT, So KF, Chang RC. Micro-dissection of rat brain for RNA or protein extraction from specific brain region. J Vis Exp. 2007;7:269. https://doi.org/10.3791/269. http://www.ncbi.nlm.nih.gov/pubmed/18989440

83. Poon DC, Ho YS, You R, Tse HL, Chiu K, Chang RC. PKR deficiency alters E. Coli-induced sickness behaviors but does not exacerbate neuroimmune responses or bacterial load. J Neuroinflammation. 2015;12:212. https://doi. org/10.1186/s12974-015-0433-2. http://www.ncbi.nlm.nih.gov/pubmed/ 26585788

84. Gould TD, Dao DT, Kovacsics CE. The Open Field. TEST. 2009;42:1-20. https://doi.org/10.1007/978-1-60761-303-9_1.

85. Vorhees CV, Williams MT. Morris water maze: procedures for assessing spatial and related forms of learning and memory. Nat Protoc. 2006; 1(2): 848-58. https://doi.org/10.1038/nprot.2006.116. http://www.ncbi.nlm.nih. gov/pubmed/17406317

86. Can A, Dao DT, Arad M, Terrillion CE, Piantadosi SC, Gould TD. The mouse forced swim test. J Vis Exp. 2012;59:e3638. https://doi.org/10.3791/3638. http://www.ncbi.nlm.nih.gov/pubmed/22314943

87. Can A, Dao DT, Terrillion CE, Piantadosi SC, Bhat S, Gould TD. The tail suspension test. J Vis Exp. 2012;59:e3769. https://doi.org/10.3791/3769. http://www.ncbi.nlm.nih.gov/pubmed/22315011

88. Ho YS, Yang $\mathrm{X}$, Lau JC, Hung CH, Wuwongse S, Zhang Q, et al. Endoplasmic reticulum stress induces tau pathology and forms a vicious cycle: implication in Alzheimer's disease pathogenesis. J Alzheimers Dis. 2012; 28(4):839-54. https://doi.org/10.3233/JAD-2011-111037. http://www.ncbi. nlm.nih.gov/pubmed/22101233

89. Yu MS, Leung SK, Lai SW, Che CM, Zee SY, So KF, et al. Neuroprotective effects of anti-aging oriental medicine Lycium barbarum against betaamyloid peptide neurotoxicity. Exp Gerontol. 2005;40(8-9):716-27. https:// doi.org/10.1016/j.exger.2005.06.010. http://www.ncbi.nlm.nih.gov/pubmed/ 16139464

90. Wuwongse S, Cheng SS, Wong GT, Hung CH, Zhang NQ, Ho YS, et al. Effects of corticosterone and amyloid-beta on proteins essential for synaptic function: implications for depression and Alzheimer's disease. Biochim Biophys Acta. 2013;1832(12):2245-56. https://doi.org/10.1016/j.bbadis.2013. 07.022. http://www.ncbi.nlm.nih.gov/pubmed/23928361

91. You R, Long W, Lai Z, Sha L, Wu K, Yu X, et al. Discovery of a potential antiinflammatory agent: 3-oxo-29-noroleana-1,9(11),12-trien-2,20-dicarbonitrile. J Med Chem. 2013;56(5):1984-95. https://doi.org/10.1021/jm301652t. http:// www.ncbi.nlm.nih.gov/pubmed/23373965

92. Werner L, Brauer K. Neuron types in the rat dorsal lateral geniculate nucleus identified in Nissl and deimpregnated Golgi preparations. J Hirnforsch. 1984; 25(2):121-7. http://www.ncbi.nlm.nih.gov/pubmed/6376620

\section{Ready to submit your research? Choose BMC and benefit from:}

- fast, convenient online submission

- thorough peer review by experienced researchers in your field

- rapid publication on acceptance

- support for research data, including large and complex data types

- gold Open Access which fosters wider collaboration and increased citations

- maximum visibility for your research: over $100 \mathrm{M}$ website views per year

At BMC, research is always in progress.

Learn more biomedcentral.com/submissions 